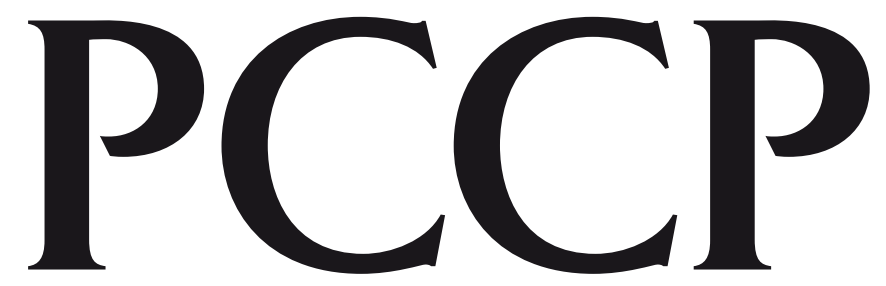

Physical Chemistry Chemical Physics

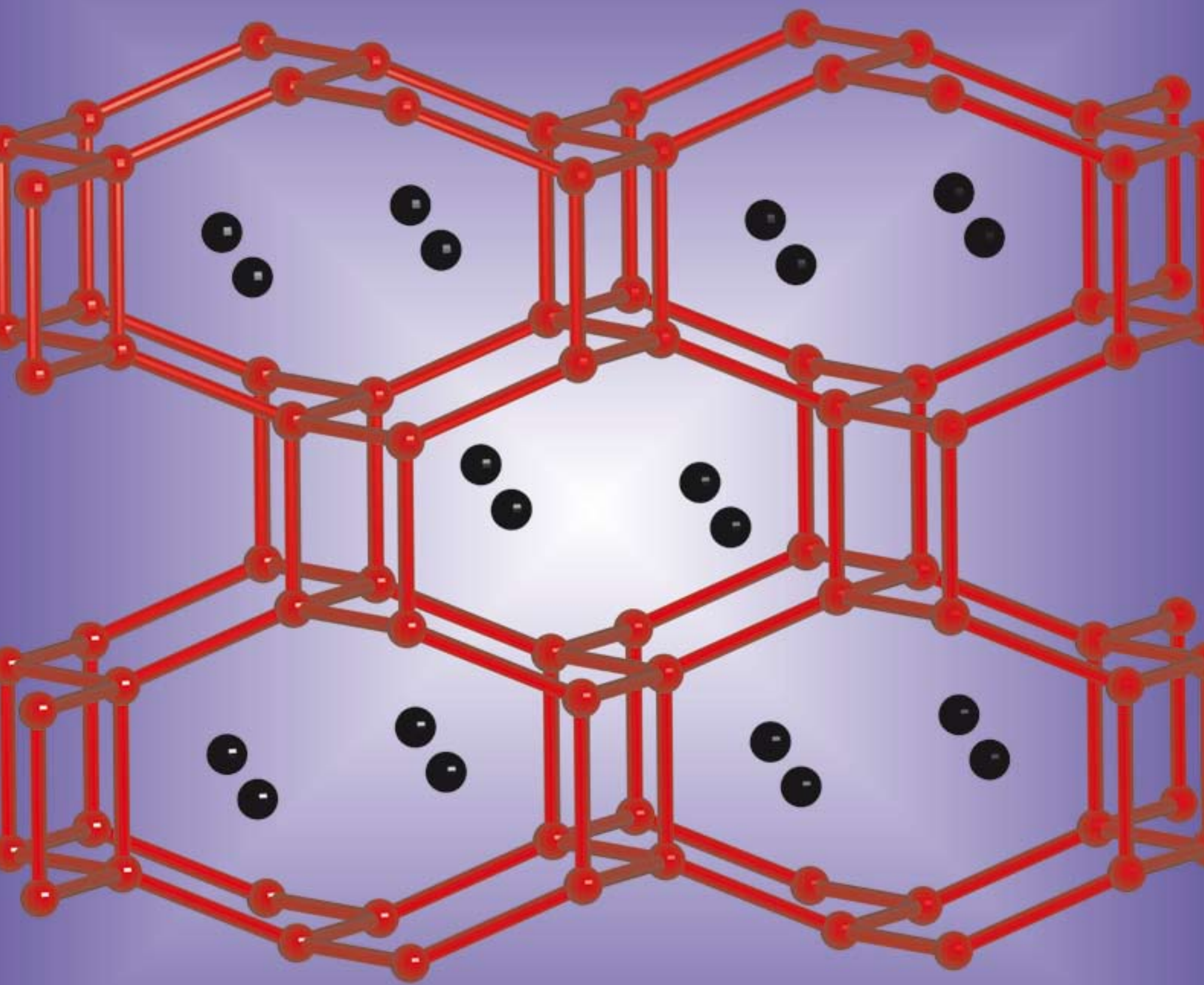

ARTICLE

Fowler et al.

Aromaticity of ring carbo-mers of [N]annulenes and [N]cycloalkanes

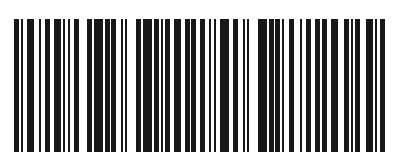

1463-9076(2008)10:7;1-6 


\title{
High-pressure gas hydrates
}

\author{
J. S. Loveday and R. J. Nelmes \\ Received 29th March 2007, Accepted 2nd July 2007 \\ First published as an Advance Article on the web 28th November 2007 \\ DOI: $10.1039 / b 704740 a$
}

It has long been known that crystalline hydrates are formed by many simple gases that do not interact strongly with water, and in most cases the gas molecules or atoms occupy 'cages' formed by a framework of water molecules. The majority of these gas hydrates adopt one of two cubic cage structures and are called clathrate hydrates. Notable exceptions are hydrogen and helium which form 'exotic' hydrates with structures based on ice structures, rather than clathrate hydrates, even at low pressures. Clathrate hydrates have been extensively studied because they occur widely in nature, have important industrial applications, and provide insight into water-guest hydrophobic interactions. Until recently, the expectation-based on calculations - had been that all clathrate hydrates were dissociated into ice and gas by the application of pressures of $1 \mathrm{GPa}$ or so. However, over the past five years, studies have shown that this view is incorrect. Instead, all the systems so far studied undergo structural rearrangement to other, new types of hydrate structure that remain stable to much higher pressures than had been thought possible. In this paper we review work on gas hydrates at pressures above $0.5 \mathrm{GPa}$, identify common trends in transformations and structures, and note areas of uncertainty where further work is needed.

\section{Introduction}

The phenomenon of water forming stable crystalline structures with gas species with which it does not interact strongly is not a new discovery: the first example of a gas clathrate hydrate - chlorine hydrate - was discovered by Davy in 1811. ${ }^{1}$ Since then, many clathrate hydrates have been found involving most simple gases (for example, nitrogen, oxygen, argon, methane) as well as larger molecules (for example, THF tetrahydrafuran). All these systems have a common structural

SUPA, School of Physics and Centre for Science at Extreme Conditions, University of Edinburgh, Mayfield Road, Edinburgh, UK EH9 $3 J Z$

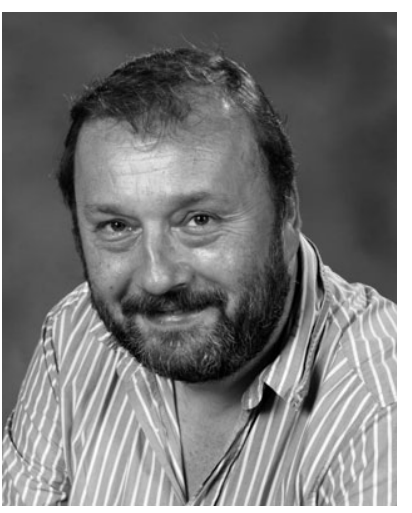

John Loveday graduated from the University of Bristol where he also took his PhD in Physics, supervised by Don Gugan. He then moved to the University of Edinburgh in 1987 to carry out post-doctoral work with Richard Nelmes, and was one of the founder members of the Paris-Edinburgh high-pressure neutron diffraction collaboration. He has specialised in techniques for high-pressure neutron scattering, and their application in studies of structures and transitions in planetary ices, hydrates, water and other simple molecular systems. In 2004, he was appointed a Reader in the School of Physics and the recently established Centre for Science at Extreme Conditions. motif in that the gas molecules (the 'guest') occupy 'host' cages formed of hydrogen-bonded water molecules, like those shown in Fig. 1. These structures appear to be stabilised by the interplay of (i) the attractions between water molecules in the cages and (ii) the repulsive hydrophobic interaction between the host and guest species. The importance of repulsions to clathrate stability can be seen from the fact that neither water itself nor ammonia, which readily forms hydrogen bonds to water, adopt these cage structures in spite of the fact that both ammonia and water have sizes comparable with those of clathrate-forming guests. Furthermore calculations show that the empty cage structure is not stable. ${ }^{2}$

Dissociation behaviour typical of gas clathrate hydrates is shown by methane hydrate. Its dissociation temperature rises

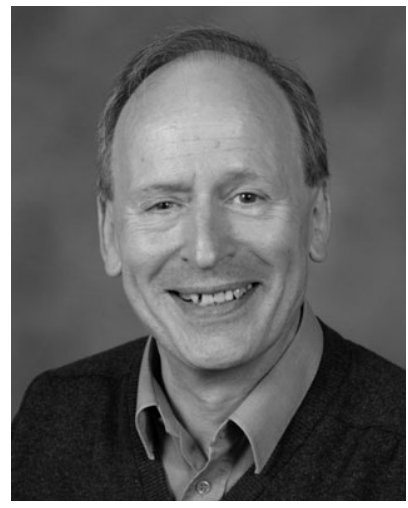

Richard Nelmes graduated from the University of Cambridge, and then took his $P h D$ in Physics at the University of Edinburgh under the supervision of Bill Cochran, with whom he did postdoctoral work until appointment as a Lecturer in 1976. After nearly 20 years of research in the field of ferroelectric structures and phase transitions, he used a Senior Fellowship from SERC (now EPSRC) to move into a specialisation in high-pressure structural physics, and his group at Edinburgh has developed leading techniques for both neutron and X-ray synchrotron diffraction. He is now Chairman of the recently established Centre for Science at Extreme Conditions in Edinburgh. 


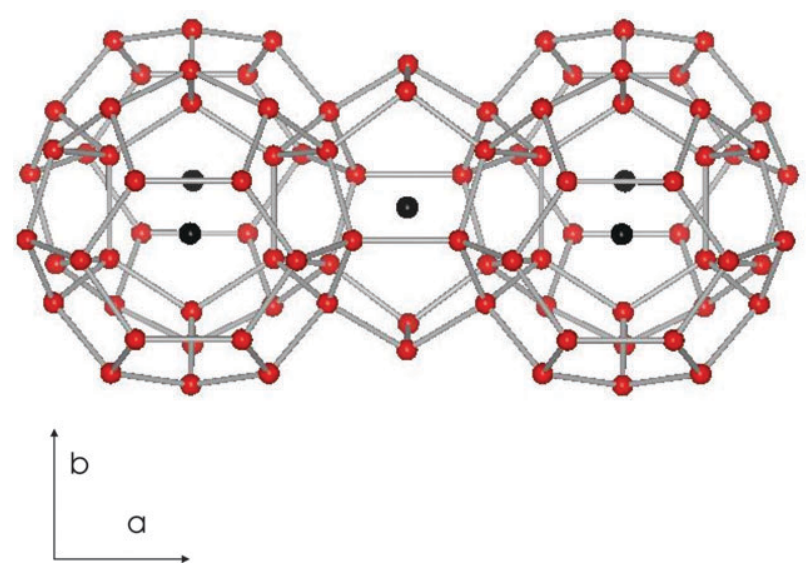

Fig. 1 The structure of cubic structure I (CS-I) clathrate hydrate. The red balls represent the oxygen atoms of the host water molecules and the black balls mark the centres of the guest molecules. For clarity the hydrogen atoms have been omitted. The crystallographic axes $a$ and $b$ are shown.

rapidly with increasing pressure in the range $0-0.5 \mathrm{GPa},{ }^{3}$ from $194 \mathrm{~K}$ at ambient pressure ${ }^{4}$ to $320 \mathrm{~K}$ (Fig. 2), and this variation can be modelled using the van der Waals-Platteuw formalism ${ }^{5}$ (see, for example, the work of Lunine and Stevenson on methane hydrate ${ }^{6}$ ). The initial rapid rise is the result of the fact that the molar volume of the clathrate is much smaller than the equivalent volume of ice (or water) and gas at low pressure. As the pressure increases, the magnitude of this volume difference, $\Delta V$, reduces since the gas is much more compressible than water or clathrate and, hence, the rate of increase of dissociation temperature with respect to pressure falls. Eventually, at yet higher pressures, the dissociated methane-water mixture becomes denser than clathrate and so $\Delta V$ passes through zero and becomes negative. Hence the dissociation temperature passes through a maximum and then falls slowly with pressure, as seen in the first part of the

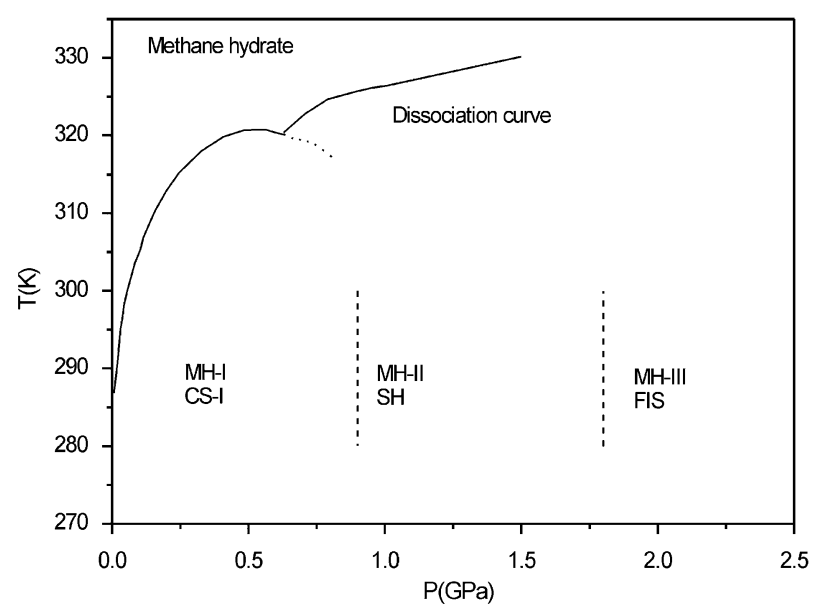

Fig. 2 The phase diagram of methane hydrate. The solid line shows the dissociation curve and the dotted line shows the initial part of the phase boundary between the two hydrate phases determined by Dyadin et al. ${ }^{3}$ The vertical dashed lines denote the transition pressures observed in our work at ambient temperature. ${ }^{35}$ Structure types are identified and the notation 'CS-I' etc. is explained in section 2. dissociation curve in Fig. 2. The rate of change is given by $\mathrm{d} T_{\mathrm{d}} / \mathrm{d} P=\Delta V / \Delta S$. At the point where the dissociation temperature, $T_{\mathrm{d}}$, starts to fall below the melting temperature of ice $(1.25 \mathrm{GPa}$ and $305 \mathrm{~K})$, the entropy change, $\Delta S$, becomes much smaller because under these conditions the clathrate would be formed from ice rather than liquid water. As a result $\mathrm{d} T_{\mathrm{d}} / \mathrm{d} P$ becomes very large and the dissociation temperature is thus calculated to fall steeply to $0 \mathrm{~K}$ (not shown in Fig. 2).

Although this conclusion is not exhaustive, in that the stability of other hypothetical hydrate structures with respect to methane and water was not tested, this model of clathrate formation and stability describes the low-pressure behaviour well, and was believed to apply to all clathrate hydrates. Hence it was concluded that pressures of around $1 \mathrm{GPa}$ (where the ice melting line reaches room temperature), or a little higher, would render all clathrate hydrates unstable at all temperatures with respect to ice and the guest species.

Pressure induced stability enhancement-i.e. the initially positive $\mathrm{d} T_{\mathrm{d}} / \mathrm{d} P$ - is a common feature of the behaviour of gas clathrate hydrates and leads to maximum stability temperatures of $10{ }^{\circ} \mathrm{C}$ or more at pressures of the order of $0.1 \mathrm{GPa}$, significantly higher than the melting point of ice. This, and the fact that clathrate hydrates form from common gases at easily accessible pressures, mean that they are widely found in nature and have important potential applications. Beds of methane hydrate at or under the ocean floor are believed to account for $30 \%$ of the Earth's methane budget and constitute an important resource. ${ }^{7}$ And, since methane is a greenhouse gas, the stability of these beds has major potential impact on the Earth's climate. ${ }^{8}$ Air hydrates in the Greenland and Antarctic ice sheets capture samples of ancient atmospheres and provide a window on the Earth's climatic history. ${ }^{9-13}$ Carbon dioxide hydrate is a potential means to sequester carbon, ${ }^{14}$ and methane hydrate is of considerable interest to the oil and gas industry both as a potential gas-transport medium and in the avoidance of pipeline blockages. ${ }^{14,15}$ Because of these important properties and applications, gas clathrate hydrates have long been extensively studied and explored in the relatively low-pressure range $(0-0.5 \mathrm{GPa}){ }^{16}$

However, the behaviour at pressures beyond this range, into the region of predicted dissociation, has been explored only in the last decade. The one exception has been in the hydrogenwater system where a monohydrate was reported in 1993 with stability up to at least $40 \mathrm{GPa},{ }^{17}$ but conventional cage clathrates were discovered in the hydrogen-water system only in $2002^{18}$ and so this example remained as an oddity. In the late 1990's, Dyadin and co-workers started a series of measurements of dissociation curves in several gas-water systems up to pressures of $1.5 \mathrm{GPa}^{3,19-23}$ In many of the systems, evidence was found of quadruple points (suggesting that new hydrate structures existed). More recent microscopic investigations ${ }^{24-46}$ have demonstrated the existence of new hydrate structures in a range of systems and in some cases gas hydrates have been found to be stable to pressures up to at least $90 \mathrm{GPa}^{26}$

This work has substantially altered the view of gas hydrates as a high-pressure phenomenon. With the single exception of hydrogen hydrate, gas hydrates had previously been viewed as a feature of relatively low pressures. The fact that gas hydrates 
are now known to persist in some cases to $\sim 100 \mathrm{GPa}$, and in general up to $\sim 10 \mathrm{GPa}$, means that they are a significant feature of the high-pressure landscape.

Here we aim to review this new work above $\sim 0.5 \mathrm{GPa}$. Work in the low-pressure range has been extensively reviewed elsewhere, for example by Sloan, ${ }^{16}$ and in the volume published by the New York Academy of Sciences, ${ }^{47}$ and we do not aim to duplicate this. However, we do include an outline of low-pressure behaviour where relevant to presenting the overall picture. A particular case is that of helium hydrate. Almost no work has been done on it above $0.5 \mathrm{GPa}$ as yet, but the lower-pressure behaviour is closely related to that of hydrogen hydrates above $2 \mathrm{GPa}$.

\section{Gas hydrate structures and notation}

Before turning to the individual systems, we note the scheme of notation to be used for the various hydrate structures encountered. At low pressures, the vast majority of the gas hydrates adopt one of two cubic clathrate structures. Cubic structure I (CS-I) in space group $P m 3 n$ has two types of cage $^{48,49}$ (see Fig. 1 and Table 1). Each unit cell contains two small cages composed of pentagons of hydrogen-bonded water molecules and six large cages composed of hexagons and pentagons. These cages are formed from forty-six water molecules and so, with one guest in each cage, CS-I has a water : guest ratio of $46: 8$ or $5.75: 1$. Cubic structure II (CS-II) has space group $F d 3 m$ and also has two types of cage ${ }^{48,50}$ (Fig. 3 and Table 2). In each unit cell, there are sixteen small cages formed of pentagons and eight large cages formed of hexagons and pentagons. There are 136 water molecules in these cages and, with one guest in each cage, CS-II has a water : guest ratio of $5.66: 1$.

Two other structures have been observed in a few exotic systems at low pressure, but (as we shall see) are more common at high pressure: these are the hexagonal clathrate structure (structure- $\mathrm{H}$ or $\mathrm{SH}$ ) which was first found in the dimethylpentane- $\mathrm{Xe}-\mathrm{H}_{2} \mathrm{~S}-$ water system ${ }^{51}$ and a tetragonal structure (structure-T or ST) first observed in the pinacolwater system. ${ }^{52}$ Finally, the ultimate high-pressure structure found in most systems in recent work has an H-bond network related to that of ice $\mathrm{I} h$ and will be referred to as the filled-ice structure or FIS. These last three structures are the principal subjects of this review and they will be described in detail where they emerge in the text.

Table 1 Space group, unit cell lattice parameter $(a)$, oxygen and cage centre fractional coordinates $(x, y, z)$, and multiplicities and Wyckoff positions $\left(6 c\right.$ etc.) of the sites for clathrate structure-I (CS-I). ${ }^{49}$ The values (and estimated standard deviations) are those obtained by Baumert et al. for methane clathrate hydrate at 100 bar and $280 \mathrm{~K}^{53}$

\begin{tabular}{ll}
\hline Space group & $P m 3 n$ \\
\hline Lattice parameter & $a=11.964(1) \AA$ \\
O1 & $0, \frac{1}{2}, \frac{1}{4}(6 c)$ \\
O2 & $0.183(1), 0.183(1), 0.183(1)(16 i)$ \\
O3 & $0,0.310(1), 0.123(1)(24 k)$ \\
Large cage centre & $0,0,0(2 a)$ \\
Small cage centre & $0, \frac{1}{4}, \frac{1}{2}(6 d)$ \\
\hline
\end{tabular}

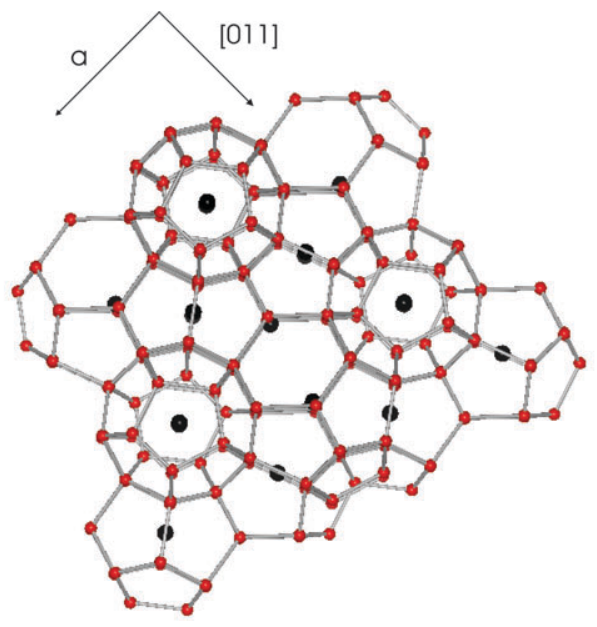

Fig. 3 The structure of cubic structure II (CS-II) clathrate hydrate viewed along a cubic [011] direction. The red balls represent the oxygen atoms of the host water molecules and the black balls mark the centres of the guest molecules. For clarity the hydrogen atoms have been omitted. The crystallographic $a$ axis and [011] direction are shown.

\section{Simple molecular hydrates}

\subsection{Methane hydrate}

Methane hydrate is the most extensively studied of all the gas hydrates. For this reason, and because much of the unexpected new behaviour at high pressure was first identified in the methane-water system, we review this system in detail and note, where appropriate, behaviour that is relevant to other gas hydrates.

Methane hydrate is common in nature and has large industrial and geoplanetary relevance. It has also been proposed as a model system for the study of the alkane-water hydrophobic interactions which are believed to play an important role in protein folding. ${ }^{55}$ And, since it is believed to have been the dominant methane-bearing phase in the nebula from which the outer planets and satellites formed, the properties of methane hydrate are crucial to models of bodies in the outer solar system. In particular, its highpressure properties are critical to understanding the origins of methane in the atmosphere of Titan, Saturn's largest moon. ${ }^{56}$

Titan is believed to have accreted from a mixture of rock, methane hydrate and ammonia monohydrate at around $120 \mathrm{~K} .{ }^{56}$ At the end of accretion, Titan would then have

Table 2 Space group, unit cell lattice parameter $(a)$, oxygen and cage fractional coordinates $(x, y, z)$, and multiplicities and Wyckoff positions $\left(8 a\right.$ etc.) of the sites for the clathrate structure II (CS-II). ${ }^{48}$ The values shown were obtained for tetrahydropyran heptadecahydrate clathrate at $230 \mathrm{~K}$ and ambient pressure by Udachin et al. ${ }^{54}$

\begin{tabular}{ll}
\hline Space group & $F d 3 m$ \\
\hline Lattice parameter & $a=17.315(7) \AA$ \\
O1 & $0,0,0(8 a)$ \\
O2 & $0.21641(1), 0.53359(1), 0.03359(1)(32 e)$ \\
O3 & $0.38076(1), 0.18224(1), 0.06776(1)(96 g)$ \\
Large cage centre & $\frac{1}{2}, \frac{1}{2}, \frac{1}{2}(8 b)$ \\
Small cage centre & $1 / 8,1 / 8,1 / 8(16 c)$ \\
\hline
\end{tabular}


consisted of an undifferentiated core made of rock plus hydrates of methane and ammonia-spanning pressures of 2-6 GPa - overlaid by a rocky carapace which was in turn overlaid by an ammonia-water ocean. ${ }^{56}$ Shortly after accretion finished, the carapace would have ruptured as the primordial core warmed and expanded through radiogenic heating, thus allowing the (lower density) icy components of the core to rise. ${ }^{56}$ Because of the high pressure of the core, the methane hydrate was assumed to have dissociated into ice and methane, according to the understanding outlined above. In that case, the released methane would have risen rapidly and escaped to the atmosphere early in Titan's history. Since solar radiation removes methane from the atmosphere by photodissociation on a timescale that is short compared to Titan's life, a surface methane repository (possibly a methane ocean) then had to be proposed to explain the known continued presence of methane in Titan's atmosphere. ${ }^{56} \mathrm{~A}$ different picture has emerged following the discovery that methane hydrate does not dissociate at these pressures.

The first evidence of new hydrates in the methane-water system came from Dyadin et al.'s differential thermal analysis (DTA) measurements ${ }^{3}$ of the decomposition line shown in Fig. 2. These show good agreement with the expected behaviour at low pressures but an increasing discrepancy at higher pressures. As well as a discontinuity in the dissociation curve at $0.62 \mathrm{GPa}$ and $\sim 318 \mathrm{~K}$ (Fig. 2), there was also evidence of a phase boundary in the solid phase, ${ }^{3}$ and Dyadin et al. concluded that this indicated a transformation from the lowpressure methane hydrate phase I (MH-I), which adopts the CS-I structure, to a higher pressure and therefore denser methane hydrate phase. Their technique allowed them to estimate the density and the value they obtained was not consistent with that expected for cubic structure-II (CS-II) clathrate, without assuming an unreasonably small lattice parameter. Hence, they concluded that a new hydrate structure was adopted. Subsequent Raman studies, direct visual observation and X-ray studies of methane-water mixtures as a function of pressure and temperature by Chou et al. ${ }^{46}$ found evidence that a methane hydrate with the CS-II structure could be formed from the melt above $0.1 \mathrm{GPa}$. Chou et al. determined the invariant point between ice VI, CS-I, water and the new high-pressure hydrate found by Dyadin et al. ${ }^{3}$ as being at $0.84 \mathrm{GPa}$ and $289.6 \mathrm{~K}$. They also showed by single-crystal energy-dispersive X-ray diffraction that this new phase had lattice parameters consistent with the SH structure. ${ }^{46}$

Although Dyadin et al. did not explore behaviour at higher pressures, they argued that methane hydrates would become unstable with respect to methane and water/ice either at the pressure where the measured dissociation curve of the new hydrate crossed the ice melting line (estimated at 1.62$1.65 \mathrm{GPa}$ ) or at $2 \mathrm{GPa}$ where the dense two-network structure ice VII becomes stable.

The first microscopic investigations in this region were carried out by Hirai and co-workers. ${ }^{25,29}$ Using synchrotron $\mathrm{X}$-ray diffraction, and diamond-anvil cells with hydrogenous samples of methane hydrate, they concluded that MH-I decomposed progressively into methane and ice over the pressure range $0.9-2.4 \mathrm{GPa}$ at room temperature, broadly consistent with dissociation at $\sim 1 \mathrm{GPa}$. However, this model appeared to be doubtful and was inconsistent with Dyadin and co-workers' finding of a new hydrate phase.

Our own neutron diffraction studies on fully deuterated methane hydrate ${ }^{35}$ found a very different behaviour. At 0.9 GPa, the CS-I structured MH-I transformed to ice VI and a new hydrate, denoted as methane hydrate II or MH-II (Fig. 2). The appearance of ice VI indicated that the new hydrate was richer in methane than the $5.75: 1$ ratio of water:methane in the original MH-I, and we estimated a ratio of $3.5(5): 1$. On further compression (see Fig. 2), this new hydrate transformed again at $1.8 \mathrm{GPa}$ to another new phase, methane hydrate-III (MH-III). Further ice VI appeared, indicating that MH-III was even richer in methane than $\mathrm{MH}-\mathrm{II}$. This third form was found to be stable up to the maximum pressure reached (6 GPa).

Our subsequent X-ray studies of hydrogenous samples found the MH-I to MH-II transition at $0.9 \mathrm{GPa}$, but then a divergence in behaviour depending on compression rate. Quickly compressed samples decomposed into methane and ice VII at around $2.4 \mathrm{GPa}$, whereas slowly compressed samples transformed to MH-III over the course of $24 \mathrm{~h}$ at $1.9 \mathrm{GPa}$. This rate dependence suggests that the MH-II to MH-III transition is kinetically hindered and that methane and ice VII are metastable with respect to ice VII and MH-III. MH-III was found in these studies to remain stable up to at least $12 \mathrm{GPa} \cdot{ }^{35}$ Finally compression at $120 \mathrm{~K}$ showed that methane hydrate becomes amorphous at around $2 \mathrm{GPa}$ and this amorphous form turns into $\mathrm{MH}-\mathrm{III}$ on warming to room temperature. This $P-T$ path is within the range estimated to have been followed by Titan's core of rock plus hydrates during the accretion phase of Titan's evolution, leading up to core overturn. ${ }^{56}$

The transition sequence was confirmed by the Raman studies of Shimizu et al., ${ }^{45}$ and careful comparison of the patterns shown by Hirai et al..$^{25,29}$ with our data ${ }^{34}$ revealed that their data were consistent with our interpretation, and in more recent studies they find essentially the same transition sequence as we do. ${ }^{26-28,30,31}$ In particular, the 'structure B' orthorhombic phase which they reported between $\sim 1.6$ and 2.1 $\mathrm{GPa}^{29,30}$ appears to be indistinguishable from MH-III and is no longer discussed.

The diffraction patterns of MH-II could be fitted with a known clathrate form, the hexagonal clathrate structure, structure-H or $\mathrm{SH}^{34,35}$ (see Table 3) - as first indicated by the lattice parameter measurements of Chou et al. ${ }^{46}$ noted above. This structure (see Fig. 4) consists of two different types of small cage and one large cage which is the length of the unit cell along the $c$-axis. It had been found hitherto only in mixed clathrate systems where a relatively large molecule (for example, dimethylpentane) fills the large cage. ${ }^{51}$ In MH-II, the most plausible model involved placing one methane molecule in each small cage and five molecules in the large cage (one at each end and three disordered over six sites around the waist). This gives a water: methane ratio of $3.4: 1$ which is consistent with the observed amount of ice produced when MH-II is formed. ${ }^{35}$ However, the quality of the data did not allow direct refinement of the methane occupancy, and hence the exact number of methane molecules in the large cage of MH-II was not decisively determined. 
Table 3 The space group, unit cell lattice parameters ( $a$ and $c$ ), oxygen and guest fractional coordinates $(x, y, z)$, and multiplicities and Wyckoff positions $(120$ etc.) of the sites for the hexagonal clathrate structure $(\mathrm{SH})$ of $\mathrm{MH}-\mathrm{II}$. The values were obtained for ArH-II from neutron diffraction data collected at $0.76 \mathrm{GPa}$ and ambient temperature by Manakov et al. ${ }^{57,58}$

\begin{tabular}{ll}
\hline Space group & $P 6 / \mathrm{mmm}$ \\
\hline Lattice parameters & $a=11.979(1) \AA, c=9.870(1) \AA$ \\
O1 & $0.792(9), 0.585(3), 0.269(3)(120)$ \\
O2 & $2 / 3,1 / 3,0.363(6)(4 h)$ \\
O3 & $0.384(2), 0,0.135(3)(12 n)$ \\
O4 & $0.863(9), 0.725(3), \frac{1}{2}(6 m)$ \\
Guest centre 1 & $\frac{1}{2}, \frac{1}{2}, \frac{1}{2}(3 g)$ \\
Guest centre 2 & $2 / 3,1 / 3,0(2 c)$ \\
Guest centre 3 & $0,0,0.297(13)(2 e)$ \\
Guest centre 4 & $0.218(15), 0.109(20), 0(6 i)$ \\
\hline
\end{tabular}

The issue of the MH-II cage occupancies was addressed in detail by Shimizu and co-workers who carried out Raman studies of single-crystal samples grown in situ in a diamondanvil cell. ${ }^{45,59}$ Their crystals were prepared by two different $P-T$ paths. First, a sample made from a mixture of ice and methane, richer in water than the MH-I composition, was compressed at $296 \mathrm{~K} .{ }^{45}$ At $0.02 \mathrm{GPa}$, a single crystal of MH-I was observed which transformed to $\mathrm{MH}-\mathrm{II}$ at $0.9 \mathrm{GPa}$. At this pressure the $\mathrm{C}-\mathrm{H}$ stretch peak was a single feature at 2914 $\mathrm{cm}^{-1}$ which was broader than the $\mathrm{C}-\mathrm{H}$ modes in CS-I, and the authors deconvolved this peak into two modes of equal intensity. (In the later work by the same group, ${ }^{59}$ it was treated as a single peak, which seems a more plausible interpretation-see below). On increase of pressure, freezing of all of the water occurred at $1.05 \mathrm{GPa}$ and the pressure dropped to 0.81 $\mathrm{GPa}$ (presumably as a result of the volume reduction as the water froze to ice VI) without any obvious change in the spectrum. On further pressure increase, a second peak with an intensity half that of the first appeared as a shoulder at 2930 $\mathrm{cm}^{-1}$ when the pressure was stepped up from 1.2 to $1.4 \mathrm{GPa}$. In the second study, a sample was grown from a melt whose composition was not given (but see below): starting at $323 \mathrm{~K}$ and $\sim 1 \mathrm{GPa}$, a single crystal of $\mathrm{MH}-\mathrm{II}$ in water was produced at $0.94 \mathrm{GPa}$ and room temperature. ${ }^{59}$ In the region of the $\mathrm{C}-\mathrm{H}$

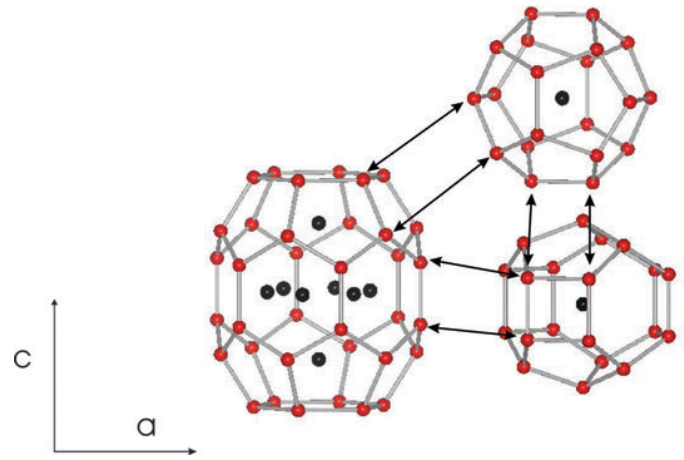

Fig. 4 Components of the hexagonal clathrate structure (SH) of $\mathrm{MH}$ II. The red and black balls represent oxygen and carbon atoms, respectively. The six sites inside the large cage that lie in a plane perpendicular to the $c$ axis are $50 \%$ occupied. For clarity the hydrogen atoms have been omitted. The arrows indicate how the cages are connected together to form a space-filling network. The crystallographic $a$ and $c$ axes are shown. stretch, a single peak was observed when MH-II initially formed at $0.94 \mathrm{GPa}$ with a width identical to that found in the first experiment. In the pressure range 1.3 to $1.4 \mathrm{GPa}$, brown patches appeared on the crystal which the authors speculated was free methane. ${ }^{59}$ (This seems unlikely. It would indicate a decrease of methane content in the samples with increasing pressure and, as discussed below, all other evidence points to the methane content increasing with pressure.) Raman spectra collected at $1.36 \mathrm{GPa}$ showed the appearance of a second $\mathrm{C}-\mathrm{H}$ peak at slightly higher frequency and with an intensity roughly a factor of two smaller, as in the first experiment. (The precise pressure at which the change occurs is unclear because no spectra were collected between 1.15 and $1.36 \mathrm{GPa}$.) At $1.4 \mathrm{GPa}$, the liquid water in the sample chamber froze, and the pressure dropped to $1.1 \mathrm{GPa}$ with the peaks remaining split. The difference in the freezing pressure in the two experiments probably indicates that the second sample was richer in methane than the first: both our $^{34}$ and Hirai et al.'s studies ${ }^{24,25}$ of the MH-I to MH-II transition started with a sample of pure MH-I and found that the water produced at the transition remained liquid to pressures of at least $1.5 \mathrm{GPa}$, and we suggested ${ }^{34}$ that this was due to the presence of traces of dissolved methane in the water. The fact that freezing occurs at lower pressures in the studies by Shimizu and co-workers, nearer the pressure expected for pure water, suggests that both their samples had more water than the MH-I composition, and by a larger amount in the first experiment. (As noted above, this behaviour is not seen in our neutron experiments with deuterated samples; MH-I transforms directly to $\mathrm{MH}-\mathrm{II}$ plus ice VI because the freezing pressure of deuterated water is below the MH-I to MH-II transition pressure.)

Hirai and co-workers also made Raman measurements ${ }^{29,30}$ and observed a single peak at $1.0 \mathrm{GPa}$ and a split peak at 1.6 GPa with the same intensity ratio as reported by Shimizu and co-workers. ${ }^{45,59}$ Chou et al. ${ }^{46}$ observed an unsplit peak at $0.88 \mathrm{GPa}$ and $298 \mathrm{~K}$ of similar width to that observed by Shimizu and co-workers, but differed in deconvolving the peak into two modes of unequal intensity. They obtained a lower frequency mode that was stronger and sharper than the higher frequency mode, with an intensity ratio of at least $4: 1$. Chou et al. did not explore to higher pressures.

Although Shimizu and co-workers described the evolution of the spectra in their first sample with pressure ${ }^{45}$ as a continuously increasing splitting of a peak which contains two modes at all pressures, examination of their data suggests that the way they interpreted their second sample ${ }^{59}$ is more plausible - that is, the first peak at $2914 \mathrm{~cm}^{-1}$ evolves continuously across the entire stability range of $\mathrm{MH}-\mathrm{II}$ and is supplemented by the appearance of a second, new $\mathrm{C}-\mathrm{H}$ stretch peak at $2930 \mathrm{~cm}^{-1}$ at a pressure between 1.20 and $1.36 \mathrm{GPa}$. This seems the most probably correct interpretation since the $2914 \mathrm{~cm}^{-1}$ peak is unchanged in width and intensity after the appearance of the second peak. Because the second peak appears at a pressure above that at which the water froze in the first sample, and a pressure below the water freezing in the second, the change appears not to be associated with the freezing of the water. In their further, most recent work, ${ }^{42}$ Shimizu and co-workers have concluded that the appearance 


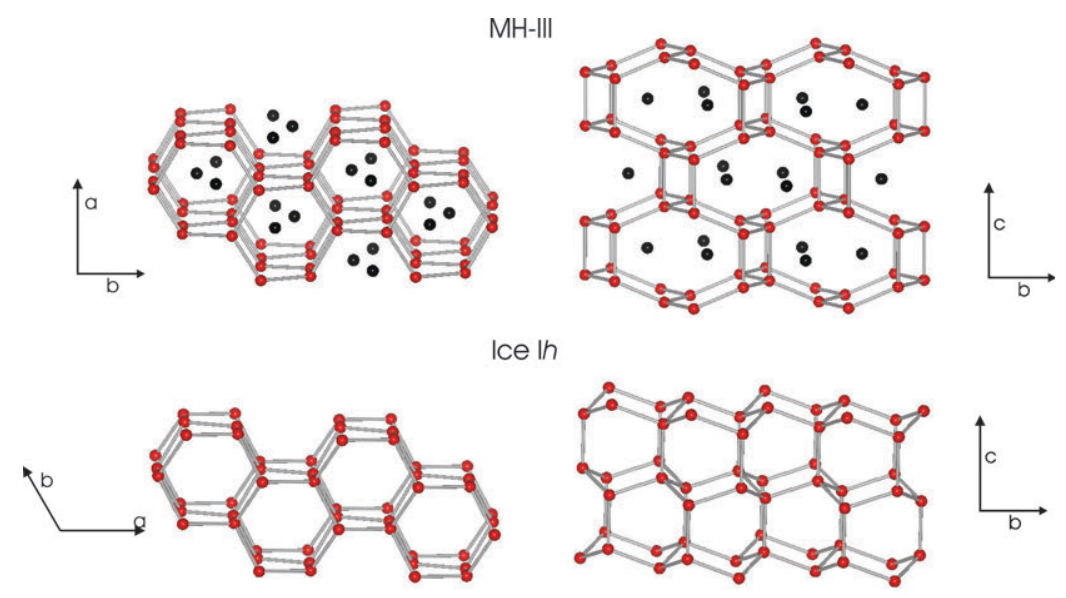

Fig. 5 The structures of MH-III (top) and ice I $h$ (bottom). The left and right hand views are, respectively, parallel and perpendicular to the hexagonal channels. The oxygen atoms are shown as red balls and the carbon atoms as black balls. For clarity the hydrogen atoms have been omitted. Crystallographic axes are shown.

of the second peak at $1.36 \mathrm{GPa}$ is the signal of a phase transition associated with a change in cage occupancy, and they denoted the higher pressure form $\mathrm{MH}-\mathrm{II}-$ prime $\left(\mathrm{MH}-\mathrm{II}^{\prime}\right){ }^{42}$ Since the unit cell of MH-II contains five of the two types of small cages (three of one type and two of the other) and a single large cage (Fig. 4), Shimizu and co-workers argued in the study of their second sample ${ }^{59}$ that placing five molecules in the large cage (with single occupancy of the five small cages) would imply roughly equal intensities for the two $\mathrm{C}-\mathrm{H}$ stretch bands, as observed in the $\mathrm{SH}$ hexagonal phase of nitrogen hydrate (see section 5.2 below). Because the small cages in $\mathrm{SH}$ have approximately the same size as the cages in CS-I, they assigned the $2914 \mathrm{~cm}^{-1}$ (higher intensity) modewhich is at roughly the same frequency as the single $\mathrm{C}-\mathrm{H}$ mode of $\mathrm{MH}-\mathrm{I}$ - to the small cages, and the new $2930 \mathrm{~cm}^{-1}$ mode to the large cages ${ }^{59}$. Hence, they argued that their $2: 1$ intensity ratio observed above $1.36 \mathrm{GPa}$ implied (i) that the large cage was not fully occupied with five molecules in $\mathrm{MH}-\mathrm{II}^{\prime}$ and (ii) that the appearance of the second higher frequency $\mathrm{C}-\mathrm{H}$ peak at $1.36 \mathrm{GPa}$ signalled an increase in occupancy of the large cages. This argument appears to imply that at pressures below $1.36 \mathrm{GPa}$, where there is the single $2914 \mathrm{~cm}^{-1}$ peak, there is only one methane environment, which would mean that either the small or large cages contain no methane. This seems implausible as it would imply that $\mathrm{MH}-\mathrm{II}$ has a $7: 1$ ratio of water: methane, which is considerably richer in water than the 5.75: 1 ratio of the parent $\mathrm{MH}-\mathrm{I}$ clathrate, and is inconsistent with the diffraction studies all of which find that excess water or ice is produced at the MH-I to MH-II transi$\operatorname{tion}^{24,25,29,30,34,35}$ so that MH-II must have somewhat less water than MH-I. In our neutron studies ${ }^{35}$ we estimated the water:methane ratio of $\mathrm{MH}-\mathrm{II}$ from the amount of ice VI produced to be $3.5(5): 1$, as said. This matches the $3.4: 1$ ratio given by 5 methane molecules in the large cages and indicates a maximum possible water: methane ratio of about $4: 1$, or an occupancy of at least 3.5 methane molecules.

It thus appears that the changes in the Raman spectra found by both Shimizu and coworkers ${ }^{42,45,59}$ and Hirai and coworkers ${ }^{24,25}$ cannot be attributed to a change in occupancy as proposed. Nonetheless, it seems well established by their work that there is a discontinuous change of some kind that none of the diffraction studies carried out to date $24,25,29,30,34,35$ shows any evidence for-neither in the hydrate structure nor in the amount of ice VI observed (in cases where the excess water is present as ice). There may be a subtle structural change that these studies have not detected, and none of them has attempted a detailed determination of the cage occupancy. It is clear that there is a need for further studies to establish the Raman mode assignments precisely, and to make more detailed diffraction studies of MH-II.

Finally, it is important to note that - as Shimizu et al. point out $^{45}$ - hydrate equilibria deal with a two-component system. All the studies to date have been carried out either at the MH-I water: methane ratio or with uncertain compositions of excess water, and no attempt has yet been made to explore the transition behaviour as a function of overall sample composition in a systematic and controlled way.

The diffraction patterns of MH-III could be indexed with an orthorhombic unit cell, and the structure was found to be that shown in Fig. 5 and detailed in Table 4 with a dihydrate composition. ${ }^{35,36}$ It is immediately obvious that the structure cannot be regarded as a cage structure. Instead, the water molecules $\mathrm{H}$-bond to form channels running along both the $a$ - and $c$-axis directions and the methane molecules are positioned within these channels (see Fig. 5). The H-bond network topology is closely related to that of ice $\mathrm{I} h$ (the ambient pressure form of ice) and differs only in the direction of one $\mathrm{H}$-bond; and so this structure is referred to as a 'filled-ice' structure (FIS) $\uparrow^{36}$ Its discovery in the methane-water system revealed the previously unknown possibility of transitions between cage clathrate and filled-ice forms. Other gas hydrates

\footnotetext{
$\dagger$ The hydrogen-water, helium-water and possibly the neon-water systems all form hydrates whose structures are examples of filled-ice structures (FIS) - based on ice Ic for hydrogen-water, and ice II for helium- and neon-water and for another phase in the hydrogen-water system. However, the MH-III (methane hydrate) structure is now commonly referred to as 'the filled-ice structure' as if it were the only one, although it is different from the others, being based on ice $\mathrm{I} h$. We draw attention to this situation, and in some places distinguish the FIS found so far in methane, argon, krypton, xenon and nitrogen hydrates as 'the MH-III filled-ice structure'.
} 
Table 4 Space group, unit cell lattice parameters ( $a, b$ and $c$ ), oxygen and carbon fractional coordinates $(x, y, z)$, and multiplicities and Wyckoff positions ( $8 i$ and $4 e$ ) of the sites for the MH-III filled ice structure. $\dagger$ The values were obtained for MH-III at $3.0 \mathrm{GPa}^{36}$

\begin{tabular}{ll}
\hline Space group & Imcm \\
\hline Lattice parameters & $a=4.7458(5) \AA \AA \AA, b=8.0644(9) \AA ⿻$ \\
$\mathrm{O}$ & $c=7.8453(7) \AA$ \\
$\mathrm{C}$ & $\frac{1}{4}, 0.4100(10), 0.1792(6)(8 i)$ \\
\hline
\end{tabular}

related to ice structures were already known: hydrogen and helium both form hydrates related to ice II, and hydrogen forms another one related to ice Ic (see the sections on hydrogen and helium hydrates below). But, since a cage clathrate was discovered in the hydrogen-water system only in $2002,{ }^{18}$ and has still not been observed in the helium-water system, no transformation between cage clathrate and filledice structures had been encountered. Following the first observation of this transition sequence in the methane-water system, it is now emerging as common to many clathrateforming systems.

Both Shimizu and co-workers and Hirai and co-workers have reported similar Raman data for MH-III. ${ }^{29,30,45}$ The $\mathrm{C}-\mathrm{H}$ stretch has a single peak, as would be expected for this structure. Interestingly, the peak is close in position (between 2-7 $\mathrm{cm}^{-1}$, depending on pressure) to the $\mathrm{C}-\mathrm{H}$ vibron of solid methane and its rate of increase with pressure is also close to that of methane. ${ }^{45}$ Hirai and co-workers found a splitting of the $\nu_{3} \mathrm{C}-\mathrm{H}$ vibron at $14 \mathrm{GPa}$ and a discontinuous softening of the $\nu_{1}$ vibron between 18 and $23 \mathrm{GPa}$ which may indicate additional intermolecular interaction around the methane molecules. However, our studies and those of Hirai and coworkers show no diffraction evidence of a structural change in this region. ${ }^{24,25,29,30,34,35}$ The phonon dispersion curves have been explored by inelastic X-ray scattering for both MH-II (at $1.7 \mathrm{GPa}$ ) and MH-III (at $2.1 \mathrm{GPa}){ }^{60}$ The experiment was complicated by the fact that the starting material used was MH-I and so the data were contaminated by scattering from excess ice VI. However, elastic and shear moduli for both hydrate phases were determined. The moduli for MH-II have values similar to those of other cage clathrates whereas the moduli of MH-III are quite different, as might be expected for this non-cage structure. ${ }^{60}$ In particular, MH-III has a stiffer elastic modulus and a much lower shear modulus than MH-II.

The stability of methane hydrate at much higher pressures has been explored by Hirai and co-workers using X-ray and Raman measurements. ${ }^{26-28}$ In a series of experiments which eventually reached a maximum pressure of $86 \mathrm{GPa}$, and included heating to $1000 \mathrm{~K}$ at this maximum pressure, ${ }^{26}$ no evidence of decomposition was observed. Instead, the disappearance of some diffraction peaks and the appearance of new peaks at $\sim 40 \mathrm{GPa}$ suggested that a further phase transition occurs at this pressure. The quality of the diffraction patterns - which contain large peaks from ice VII - did not permit solution of the structure or unambiguous indexing of the unit cell. Based simply on $\mathrm{O} \cdots \mathrm{O}$ distances estimated from the lattice parameters, and on the assumption of no change in atomic fractional co-ordinates from those determined at $3.0 \mathrm{GPa},{ }^{36}$ it was suggested that the transition may be asso- ciated with centring of the H-bonds in MH-III. However, the structural pressure dependence of MH-III has not yet been determined and it is unlikely that the fractional co-ordinates do not change with pressure. Klug and co-workers carried out infrared studies of dilute $\mathrm{H}_{2} \mathrm{O}$ in $\mathrm{D}_{2} \mathrm{O}$ methane hydrate to study the uncoupled $\mathrm{O}-\mathrm{H}$ stretch frequency up to $40 \mathrm{GPa} .{ }^{61}$ This experiment was also complicated by having MH-I as the starting material, so that the resulting MH-III sample contained excess ice. However the Fermi resonance observed in the stretch frequency differed somewhat from that observed in pure ice and - with assistance from ab initio molecular dynamics calculations - it was concluded that centring was complete by $60 \mathrm{GPa} .{ }^{61}$ Other total-energy computational studies by Iitaka and Ebisuzaki found centring starting at $40 \mathrm{GPa},{ }^{62,63}$ which supports the suggestion of Hirai and coworkers, ${ }^{27,28}$ but the structural arguments are doubtful as discussed above. A determination of the structural pressure dependence of MH-III to $40 \mathrm{GPa}$ and above is needed.

All these studies have revealed that the behaviour of methane hydrate is remarkably different from that expected. Not only does it not decompose at $\sim 1 \mathrm{GPa}$, but it remains stable to pressures almost two orders of magnitude higherat least. This gives a remarkably large variation in the C...O distances from $3.8 \AA$ in MH-I to $3.3 \AA$ at $3.0 \mathrm{GPa}$, and to perhaps less than $3.0 \AA$ by $40-50 \mathrm{GPa}$ if there is little change in the fractional co-ordinates. Methane hydrate thus provides a very good system to explore the repulsive potential over a wide range of distances. ${ }^{55}$ Moreover, the existence of a transition from cage clathrate to filled ice opens up a new possible transition path that, as we shall see, turns out to be common to many cage clathrates. Finally, the fact that methane hydrate does not decompose at pressures as low as $1 \mathrm{GPa}$ has changed the basis of modelling of Titan. ${ }^{35}$ Instead of the primordial methane all reaching the surface early in Titan's history when the originally-accreted core overturned (see above), the methane would have remained in stable hydrates - transforming back to MH-I with reducing pressure ${ }^{35}$ - and risen slowly with the other icy components of the core to produce a subsurface layer of MH-I. ${ }^{35}$ Such a layer provides a much more plausible reservoir to supply the atmosphere with methane via cryovulcanism. ${ }^{64,65}$ It is exceedingly unlikely that the rate of outgassing due to cryovulcanism is exactly equal to the rate of methane loss by photo-decomposition. And a rate of outgassing greater than the rate of loss would account for the surface pools of methane recently reported. ${ }^{66}$

\subsection{Other simple molecular hydrates}

Only two other systems with polyatomic molecular guests have been studied to any significant extent to higher pressures, namely, tetrahydrofuran (THF) clathrate and sulfur hexafluoride $\left(\mathrm{SF}_{6}\right)$ clathrate. Manakov et al. have found two new hydrates in the THF-water system above 0.25 and $0.49 \mathrm{GPa}$, respectively, and dissociation into THF and water at $3 \mathrm{GPa},{ }^{67}$ but there is to date no structural characterisation. Aladko et $a l .{ }^{68}$ and Dyadin et al. ${ }^{69}$ have studied the dissociation curve of the $\mathrm{SF}_{6}$-water system using DTA and found evidence for two new hydrates. Using X-ray diffraction techniques, they showed that the first transition at $0.05 \mathrm{GPa}$ is a change from 
CS-II to CS-I. The second transition at $0.13 \mathrm{GPa}$ is isostructural and involves population of the small cage with guest species, as shown by neutron diffraction studies at $0.9 \mathrm{GPa}^{68,69}$ Based on Raman data they argued that no further structural transitions occur at higher pressures but found evidence for dissociation into ice and $\mathrm{SF}_{6}$ at $4.4 \mathrm{GPa}^{68,69}$

There has also been work on these hydrates at low temperatures $(77 \mathrm{~K})$. Handa et al. found some time ago that both systems undergo a volume collapse at $\sim 1.6 \mathrm{GPa}^{70}$ The behaviour appeared very similar to that observed in ice ${ }^{71}$ and hence the collapse was interpreted as pressure-induced amorphisation. This view was supported by modelling stu$\operatorname{dies}^{72-74}$ and subsequently confirmed by neutron diffraction studies on THF hydrate 37,75 - and similar behaviour was found in methane hydrate. ${ }^{35}$ Surprisingly, unlike the amorphisation of ice, the transition in the THF and $\mathrm{SF}_{6}$ hydrates is reversible $\mathrm{e}^{70}$ and the crystalline clathrate may be reformed on decompression. Modelling studies suggested that the reversibility is due to the presence of guest atoms within the amorphised ice lattice, which provides a "spring" to restore the original structure. ${ }^{73}$ A detailed structural investigation of this behaviour has not yet been carried out.

$\mathrm{CO}_{2}, \mathrm{CO}$ and $\mathrm{H}_{2} \mathrm{~S}$ hydrates would also be of considerable interest but have not yet been extensively studied. The dissociation curve of $\mathrm{CO}_{2}$ hydrate has been determined by Dyadin et al. ${ }^{76}$ and evidence for a second hydrate has been found above $0.6 \mathrm{GPa}$, but this has not been verified by microscopic investigations. CO hydrate ${ }^{77}$ would be of interest since $\mathrm{CO}$ is isoelectronic with $\mathrm{N}_{2}$ and a comparative study with $\mathrm{N}_{2}$ hydrate would be valuable. But to date high-pressure behaviour seems not to have been explored. $\mathrm{H}_{2} \mathrm{~S}$ hydrate ${ }^{78}$ offers the possibility of observing the onset of guest-host hydrogen bonding under pressure, but again this has yet to be explored.

\section{Noble gas hydrates}

\subsection{Helium hydrate}

The helium-water system remains relatively unexplored. Londono et al. identified the first helium hydrate using neutron powder diffraction at pressures between 0.28 and $0.5 \mathrm{GPa}^{79}$ The structure is a filled-ice structure based on that of ice II (Fig. 6 and Table 5). The rhombohedral ice II structure has hexagonal channels running along the $c$-axis and the helium atoms sit in these channels giving a hydrate with a $6: 1$ ratio of water: helium at full helium occupancy. ${ }^{79-81}$ Studies of the phase diagram are complicated by the fact that ice II itself is stable under similar $P$ and $T$ conditions. Londono et al. ${ }^{80}$ showed that the presence of helium suppresses the formation of ices III and V and hence reports of ice II in the domains of these phases may be the result of the use of helium as a pressure medium and the misidentification of helium hydrate. The presence of helium was shown to distort the ice II structure, and expand the channels in the $a b$-plane and shorten them in the $c$-direction. ${ }^{79-81}$ In their 2002 paper, Lobban et al. ${ }^{81}$ argued that none of the in situ structural studies of

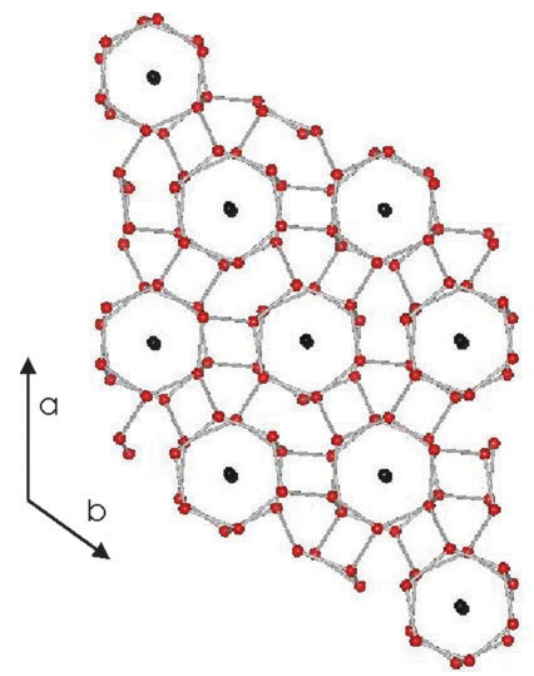

Fig. 6 The structure of helium hydrate viewed along the hexagonal $c$ axis. The red balls represent the oxygen atoms of the host water molecules and the black balls mark the centres of the guest molecules. For clarity the hydrogen atoms have been omitted. The crystallographic $a$ - and $b$-axes are shown.

ice II published prior to their work was free from the effects of partial formation of hydrate and hence was not reliable.

Diffraction studies of helium hydrate have not been taken further than 0.5 GPa, but Dyadin and co-workers explored the dissociation curve of helium hydrate up to $1.5 \mathrm{GPa}$ using DTA. $^{22}$ In addition to reproducing the behaviour reported by Londono et al., ${ }^{80}$ and extending the curve to higher pressures, they also found thermal anomalies in the 0.1-0.2 GPa range that they interpreted as the signature of a classical clathrate hydrate in the helium-water system. This conclusion remains unverified microscopically but, given the observation of a classical hydrogen clathrate (see the section on hydrogen hydrate below), it is plausible. Analogies with the hydrogen-water system, which also forms an ice-II-based hydrate, suggest that helium may form an ice-Ic-based hydrate at higher pressures, but this possibility remains to be investigated.

\subsection{Neon hydrate}

Dyadin and co-workers measured the decomposition line of neon hydrate using DTA up to $1.5 \mathrm{GPa}^{21,22}$ Above $\sim 0.25$ $\mathrm{GPa}$, the curve lies above the melting line of water and rises at roughly the same rate with pressure. Because the pressure dependence of the decomposition line was so markedly different from that of a cage clathrate (see Fig. 2), they concluded that neon forms an ice-II-based hydrate. Between 0.2 and

Table 5 The space group, unit cell parameters ( $a$ and $c$ ), oxygen and helium fractional coordinates $(x, y, z)$, and multiplicities and Wyckoff positions $(18 \mathrm{f} \mathrm{etc}$.) of the sites for helium hydrate. The values were obtained at $197 \mathrm{~K}$ and $0.291 \mathrm{GPa}$ by Londono et al. ${ }^{80}$

\begin{tabular}{ll}
\hline Space group & $R \overline{3}$ \\
\hline Lattice parameters & $a=12.921(5) \AA ̊ \AA, c=6.205(5) \AA$ \\
$\mathrm{O} 1$ & $0.2216(4), 0.1976(4), 0.0477(8)(18 f)$ \\
$\mathrm{O} 2$ & $0.1893(4), 0.2311(3), 0.4801(9)(18 f)$ \\
$\mathrm{He}$ & $0,0,0.297(6)(6 c)$ \\
\hline
\end{tabular}


$0.3 \mathrm{GPa}$ they also found evidence for formation of a classical clathrate hydrate with slow formation kinetics. ${ }^{21,22}$ Neither of these findings has been investigated microscopically.

\subsection{Argon hydrate}

The argon-water system is of interest because the interaction potential between argon and water is relatively simple to describe and hence it is tractable to modelling. At low pressures, a cubic structure II (CS-II) cage clathrate is formed, argon hydrate I (ArH-I). As with other gas cage-clathrates, the decomposition curve rises steeply in the low-pressure region. At higher pressures the dissociation curve has been explored by DTA to $1.5 \mathrm{GPa}^{20,57}$ and by Raman scattering to $3 \mathrm{GPa}^{82}$ (Fig. 7). The studies are in agreement with each other and find clear evidence of the existence of three new hydrates. Manakov and co-workers published the first diffraction data on this system using neutron powder techniques. ${ }^{57}$ At ambient temperature, ArH-I transformed at $0.46 \mathrm{GPa}$ to ArH-II which they found has the SH structure. At $0.77 \mathrm{GPa}$, ArH-II transformed to ArH-III whose structure they suggested was ice-II-based. This suggestion was corrected in a later study ${ }^{58}$ which showed that it was in fact a tetragonal structure (structure-T or ST) previously only seen in the pinacol semiclathrate. ${ }^{52}$ The structure is shown in Fig. 8 and details are given in Table 6. Two argon atoms occupy a single type of cage, ${ }^{83}$ and this is the only gas-hydrate structure with a single cage type. Hirai et al. have carried out X-ray diffraction studies, ${ }^{31,32}$ and confirmed the formation of the tetragonal structure of ArH-III at $\sim 0.7 \mathrm{GPa}$ but missed the formation of the hexagonal form ArH-II. They also identified a further transition at $1.1 \mathrm{GPa}$ at which the filled-ice structure is formed (ArH-IV). This structure was found to decompose at $6.1 \mathrm{GPa}$ into ice and argon. ${ }^{31,32}$ Our own studies using neutron diffraction confirmed this transition sequence. ${ }^{38}$ More recently, Manakov and coworkers have published a more detailed

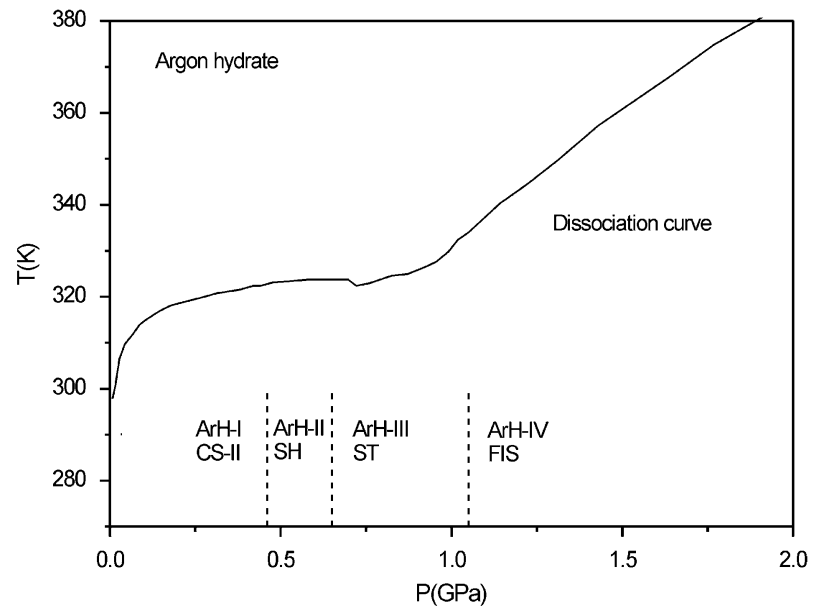

Fig. 7 Phase diagram of argon hydrate. The solid line is the dissociation curve taken from Manakov et al. ${ }^{57}$ and is a compendium of data obtained in this study along with data from Marschall et al.,${ }^{84}$ Dyadin et al. ${ }^{20}$ and Lotz and Schouten. ${ }^{82}$ The vertical dashed lines denote the pressures observed for the various solid-solid phase transitions at ambient temperature. Structure types are identified, and the notation 'CS-II' etc. is explained in section 2.

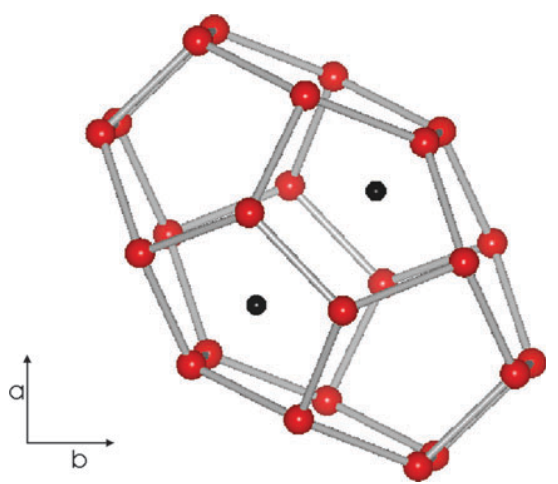

Fig. 8 One cage of the tetragonal structure (ST) of argon hydrate phase III, ArH-III. The red balls represent oxygen atoms and the black balls represent argon atoms. For clarity, the hydrogen atoms have been omitted. The crystallographic $a$ and $b$ axes are shown.

diffraction study of ArH-II and ArH-III, which, as discussed below, included a refinement of the cage occupancies. ${ }^{58}$

Shimizu et al. measured the Raman spectra of all four phases of argon hydrate and obtained transition pressures consistent with those given above. ${ }^{43}$ In the first three phases, they observed a low-energy mode at $\sim 130 \mathrm{~cm}^{-1}$. Based on the fact that neither ice nor the CS-II nitrogen hydrate has modes in this region, and supported by previous calculations of the Raman spectra of multiply-occupied cages ${ }^{85}$ they argued that the mode corresponded to $\mathrm{Ar}-\mathrm{Ar}$ interatomic stretching vibrations in doubly occupied cages. This would indicate at least partial double occupancy of the cages in the first three phases of argon hydrate. However, the group's subsequent study of krypton hydrate showed a mode at a similar frequency ${ }^{40}$ Since a $\mathrm{Kr}-\mathrm{Kr}$ vibration should have quite different frequencies from an $\mathrm{Ar}-\mathrm{Ar}$ vibration, because of the difference in atomic mass, they concluded that they had wrongly interpreted the $130 \mathrm{~cm}^{-1}$ mode as arising from guest-guest vibrations in doubly occupied cages, and that they must instead be modes involving the oxygen atoms. ${ }^{40}$ It thus appears that Raman studies are so far essentially silent on the matter of cage occupancies in the rare-gas hydrates. Shimizu et al. also noted that the Raman spectrum of ArH-IV was like that observed for the ring structure of ice $\mathrm{II}^{43}$ but since the filled-ice structure also has six-fold rings, their data are equally consistent with the conclusion from diffraction studies that ArH-IV has that structure. ${ }^{31,38}$

Meanwhile, Manakov et al. were able to carry out structure refinements of the argon occupancy in the first three hydrate phases. ${ }^{58}$ In ArH-I, the occupancy of the large cage refined to a value between 1.8 and 2.2 depending on pressure. In ArH-II

Table 6 The space group, unit cell lattice parameters ( $a$ and $c$ ), oxygen and argon fractional coordinates $(x, y, z)$, and multiplicities and Wyckoff positions of the sites for the structure-T (ST) hydrate structure. The values were obtained for ArH-III from neutron diffraction data at $0.9 \mathrm{GPa}$ and ambient temperature by Manakov et al. ${ }^{57,58}$

\begin{tabular}{ll}
\hline Space group & $P 4_{2} / m n m$ \\
\hline Lattice parameters & $a=6.342(2) \AA ⿻ 一 丿, c=10.610(3) \AA$ \\
$\mathrm{O} 1$ & $0, \frac{1}{2}, \frac{1}{4}(4 d)$ \\
$\mathrm{O} 2$ & $-0.147(3),-0.147(3), 0.643(3)(8 j)$ \\
$\mathrm{Ar}$ & $0.181(5), 0.181(5), 0(4 f)$ \\
\hline
\end{tabular}


they found a five-fold occupancy of the large cage in line with the structural model originally proposed for MH-II. ${ }^{34}$ And in ArH-III they confirmed the double occupancy of the single cage, although they do not appear to have refined the occupancies of the small cages in either ArH-I or ArH-III. Recently, modelling studies have looked at the stability of all the noble gas hydrates with the MH-II structure. ${ }^{86}$ For argon hydrate, the results show that the lowest free energy occurs for an occupancy of five argon atoms in the large cage.

\subsection{Krypton hydrate}

Krypton, like argon, is a computationally tractable guest species and since it is larger it provides a means to explore the effect of guest size. The low-pressure form $(\mathrm{KrH}-\mathrm{I})$ has the CS-II structure. Dyadin et al. have measured the dissociation curve of the krypton-water system using DTA and found evidence of three high-pressure hydrates up to $1.5 \mathrm{GPa}^{19}$ (Fig. 9). Synchrotron X-ray diffraction studies have been carried out by Desgreniers and co-workers. ${ }^{87}$ They found a transformation from CS-II (KrH-I) to CS-I (KrH-II) at 0.45 $\mathrm{GPa}$, a further transformation at $0.75 \mathrm{GPa}$ to structure-H (KrH-III) and then formation of the MH-III filled-ice structure (KrH-IV) at $1.8 \mathrm{GPa}$. This last form then decomposed into ice and krypton at 2.7-2.8 GPa. Sasaki et al. measured the Raman spectra of this system and obtained the same transition pressures $^{40}$ within the precision of the measurements $( \pm 0.1 \mathrm{GPa})$. As mentioned in section 4.3 , the $\sim 130 \mathrm{~cm}^{-1}$ mode which Shimizu et al. observed in $\mathrm{ArH}-\mathrm{II}^{43}$ was found at almost the same frequency in $\mathrm{KrH}-\mathrm{I}$ and $\mathrm{KrH}-\mathrm{III}$, but disappeared in the CS-I structured $\mathrm{KrH}_{-} \mathrm{II} .{ }^{40}$ And the lack of change in frequency on going from argon to heavier krypton indicated that this mode could not be assigned to guest-guest vibrations and must, instead, be associated with the oxygen network. Based on (i) visual observation of the sample, in

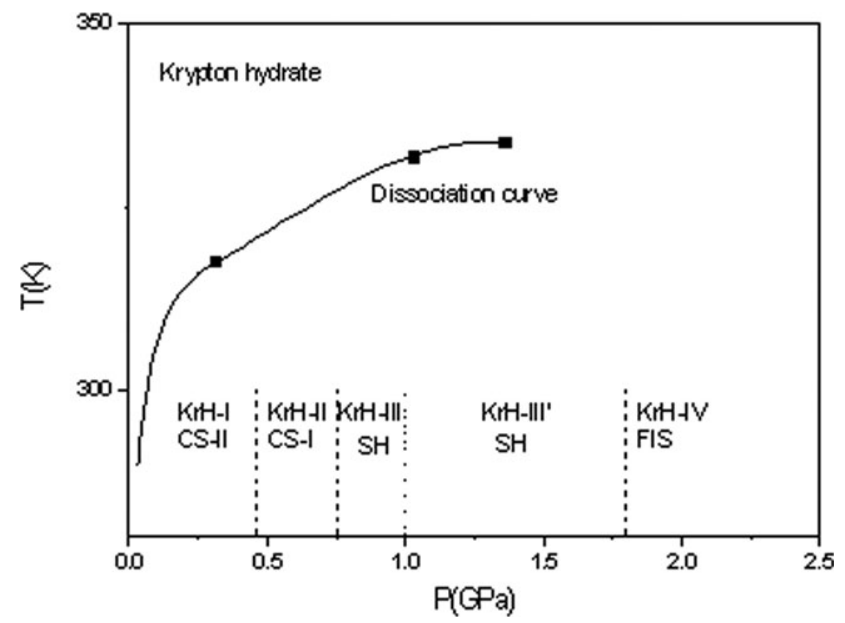

Fig. 9 The phase diagram of krypton hydrate. The solid line shows the dissociation curve taken from Dyadin et al. ${ }^{19}$ and the squares mark the positions of the three quadruple points found in this work. The vertical dashed lines denote the pressures of the structural phase transitions observed at ambient temperature and the vertical dotted line at $1 \mathrm{GPa}$ shows the occupancy transition proposed by Sasaki et $a l .{ }^{40}$ Structure types are identified, and the notation 'CS-II' etc. is explained in section 2 . which spots of what appeared to be free water were observed, and (ii) on a jump in the $\mathrm{O}-\mathrm{H}$ vibron frequency, they proposed a further transition in the $\mathrm{KrH}-\mathrm{III}$ field at $1.0 \mathrm{GPa}$ to $\mathrm{KrH}-$ III' $^{\prime}$. Because of the appearance of water, Sasaki et al. ${ }^{40}$ argued that this transition involves an increase in the large-cage occupancy rather than a change in crystal structure. Some support for this suggestion comes from the modelling work of Alavi et al. ${ }^{86}$ They find the optimal occupancy of the large cage to be three krypton atoms at both ambient pressure and $2 \mathrm{GPa}$, and conclude that an occupancy of four remains unstable but becomes less so with increasing pressure, which suggests that pressure tends to stabilise higher occupancy. Clearly, as is the case for MH-II, a determination of the cage occupancy in this pressure range is needed and requires detailed diffraction studies.

Sasaki et al. also found a significant difference between the $\mathrm{O}-\mathrm{H}$ stretch frequencies of the filled-ice structured forms of argon and krypton hydrate (ArH-IV and KrH-IV). This suggests that the $\mathrm{H}$-bond length in the filled-ice structure of these phases is determined by the size of the guest and is expanded in $\mathrm{Kr}-\mathrm{VI}$ relative to ArH-IV. ${ }^{40}$ Sasaki et al. did not observe decomposition of $\mathrm{KrH}-\mathrm{IV}$ up to a maximum pressure of almost 5.2 GPa, nearly twice as high as the decomposition pressure found by Desgreniers. ${ }^{87}$ Further work is needed to explore the effects of composition and $P-T$ path to settle this apparent discrepancy.

\subsection{Xenon hydrate}

At low pressures, xenon hydrate-I adopts the CS-I structure. Dyadin and co-workers found no evidence of a new hydrate in the xenon-water system up to $1.5 \mathrm{GPa}$ in DTA studies. ${ }^{19}$ The first diffraction data on this system were collected by Sanloup et al. using energy-dispersive methods. ${ }^{88}$ They found a phase transition at $1.8 \mathrm{GPa}$ from the CS-I structure to a new phase (XeH-II) which they indexed as tetragonal. Based on the ratio of the xenon fluorescence to the strongest diffraction peak they argued that $\mathrm{XeH}-\mathrm{II}$ has the same $5.75: 1$ ratio of water: xenon as XeH-I. ${ }^{88}$ On further pressure increase the sample decomposed at 2.9 GPa. Our X-ray and neutron studies revealed that this XeH-II in fact adopts the $\mathrm{SH}$ clathrate structure and the appearance of either water or ice at the transition indicated that it was richer in xenon than the parent phase. ${ }^{38}$ The modelling work of Alavi et al. ${ }^{86}$ finds the optimal occupancy for the large cage to be two xenon atoms, which implies a composition of $4.86: 1 .^{86}$ It thus appears that although XeH-II is richer in xenon than XeH-I, the larger size of the xenon atom may result in a smaller occupancy of the large cage than the five found for ArH-II and proposed for MH-II. Our studies also confirmed the decomposition of xenon hydrate into ice and xenon at $2.9 \mathrm{GPa}^{38}$

\section{Diatomic element hydrates}

\subsection{Hydrogen hydrate}

The phase relations in the hydrogen-water system were first explored by Vos et al. ${ }^{17}$ Using a combination of visual observation and light-scattering techniques, they were able to measure the dissociation curve, which lies above the melting 
Table 7 The space group, unit cell lattice parameter (a), oxygen and hydrogen (centre of molecule) fractional coordinates $(x, y, z)$, and multiplicities and Wyckoff positions $(4 a$ and $4 b$ ) of the sites for the $\mathrm{C} 2$ hydrogen clathrate as determined at $3.1 \mathrm{GPa}$ and room temperature ${ }^{17}$

\begin{tabular}{ll}
\hline Spacegroup & $F d 3 m$ \\
\hline Lattice parameter & $a=6.434(1) \AA$ \\
$\mathrm{O}$ & $0,0,0(4 a)$ \\
Hydrogen centre & $\frac{1}{2}, \frac{1}{2}, \frac{1}{2}(4 b)$ \\
\hline
\end{tabular}

curve of ice up to the maximum pressure studied (3.5 GPa). At 2.3 and $3.1 \mathrm{GPa}$, they observed quadruple points which correspond to the upper and lower bounds of the region of coexistence between two different solid hydrate phases. X-Ray diffraction showed that the lower pressure phase (labelled $\mathrm{C} 1$ ) had a rhombohedral unit cell like that of ice II and helium hydrate (see above), ${ }^{79}$ and Raman studies yielded an estimated water: hydrogen ratio of between $10: 1$ and $5: 1$. Given the similarities in unit cells and water:guest ratios, Vos et al. suggested that the $\mathrm{C} 1$ hydrogen hydrate has a similar structure to that of helium hydrate. The higher pressure form (labelled C2) was found from single-crystal X-ray data to have a cubic unit cell and an oxygen structure like that of ice Ic (Table 7 and Fig. 10). The oxygen atoms form a tetrahedrally coordinated $\mathrm{H}$-bond network which has large voids. Vos et al. suggested that the hydrogen molecules sit in these voids giving a 1:1 water: hydrogen composition-consistent with their Raman and visual observations. ${ }^{17}$ This structure for $\mathrm{C} 2$ is closely related to that of ice VII: in ice VII there are two interpenetrating ice $\mathrm{I} c$-like networks and in the structure of $\mathrm{C} 2$ one of the networks is replaced by hydrogen. From a combined X-ray and Raman study, Vos et al. demonstrated that C2 was stable to at least $60 \mathrm{GPa}$ and they found its bulk modulus to be $\sim 30 \%$ less than that of ice VII. They found that the pressure dependence of the $\mathrm{O}-\mathrm{H}$ stretch frequency and the hydrogen bond length could be mapped onto those of ice VII simply by multiplying the pressure in the $\mathrm{C} 2$ experiments by a factor of two, and hence they concluded that the hydrogen bonds in $\mathrm{C} 2$ may centre at around $40 \mathrm{GPa} .{ }^{89}$ Dyadin and co-workers reproduced the Vos et al. dissociation curve up to $1.5 \mathrm{GPa}$ and found evidence for a classical clathrate hydrate between 0.2 and $0.4 \mathrm{GPa}^{21}$ Mao et al. recently

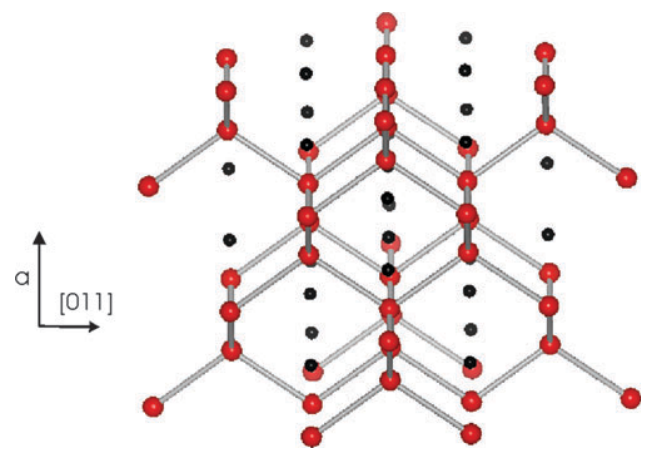

Fig. 10 The structure of $\mathrm{C} 2$ hydrogen hydrate. The red balls denote oxygen atoms and the black balls mark the centres of the hydrogen molecules. For clarity the hydrogen atoms of the water molecules have been omitted. The crystallographic $a$-axis and [011] direction are shown. confirmed this and showed it had the CS-II structure with a $2: 1$ water : hydrogen ratio. ${ }^{18}$

\subsection{Nitrogen hydrate}

Nitrogen hydrate has been quite extensively studied as a model system for the effect of pressure on cage occupancies. Kuhs and co-workers have demonstrated using neutron diffraction that occupancies of greater than one molecule per cage can be obtained and that the behaviour of the occupancy as a function of pressure can be modelled using a Langmuir-Bloggett formalism. ${ }^{10}$ The first evidence of a new hydrate structure above $0.5 \mathrm{GPa}$ in this system came from Raman studies by van Hinsberg et al. ${ }^{90}$ in which a splitting of the nitrogen vibron into two peaks was observed at $0.84 \mathrm{GPa}$ and $295 \mathrm{~K}$. Dyadin et al.'s DTA measurements of the dissociation curve (Fig. 11) identified two high-pressure hydrates, the first appearing at $\sim 0.75 \mathrm{GPa}$ and the other at $\sim 1.2 \mathrm{GPa}^{23}$ Our own neutron diffraction studies ${ }^{38}$ found the transition sequence to be from the CS-II structure (NH-I) to structure-H (NH-II) at $\sim 0.9$ $\mathrm{GPa}$, followed by a transformation to the tetragonal structure$\mathrm{T}(\mathrm{NH}-\mathrm{III})^{83}$ at $\sim 1.6 \mathrm{GPa}$, and then to the filled-ice structure at $\sim 2 \mathrm{GPa}(\mathrm{NH}-\mathrm{IV})$.

Sasaki et al. carried out Raman studies on this system and obtained a pressure of $0.92 \mathrm{GPa}$ for the NH-I to NH-II transition, and found a further transition at $1.48 \mathrm{GPa}$ with no further transitions or dissociation up to a maximum of $6 \mathrm{GPa}^{39}$ They concluded that the transition at $1.48 \mathrm{GPa}$ was to NH-IV (filled-ice structure) and they found no evidence of NH-III. ${ }^{39}$ In the NH-II phase, they found that the N-N vibron was split into two modes of equal intensity and hence concluded that the large cage of the hexagonal structure contained five nitrogen molecules. This is in contrast to their work on methane hydrate II where a $2: 1$ intensity ratio of the $\mathrm{C}-\mathrm{H}$ vibron led them to propose an occupancy of less than five for the large cage. Nitrogen hydrate did not dissociate up to the

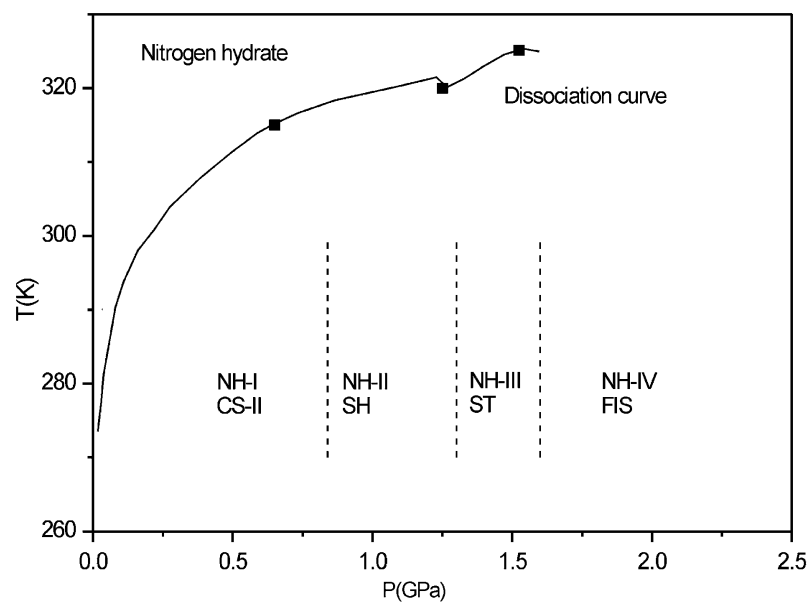

Fig. 11 The phase diagram of nitrogen hydrate. The solid line shows the dissociation curve taken from Dyadin et al. ${ }^{23}$ and is a compendium of data obtained by van Hinsberg et al. ${ }^{90}$ and Marschall et al. ${ }^{84}$ The squares mark quadruple points found by Dyadin et $a l . .^{23}$ The dashed lines denote the pressures of the solid-solid phase transitions observed at ambient temperature. Structure types are identified, and the notation 'CS-II' etc. is explained in section 2. 
maximum pressure achieved in these studies (6.0 GPa). Hirai et al. state that nitrogen hydrate dissociates at this pressure, but they do not offer either a reference or data to support this statement. ${ }^{91}$ Our unpublished studies indicate stability of the $\mathrm{NH}-\mathrm{IV}$ phase up to at least $30 \mathrm{GPa}$.

\subsection{Other diatomic element hydrates}

Chlorine hydrate was the first clathrate to be observed, as already noted above, and oxygen hydrate is of interest since the oxygen molecule is similar in size to nitrogen. However, neither of these two clathrates, nor the other halogen hydrates, have been studied at pressures above $0.5 \mathrm{GPa}$. Given the apparent difference between the behaviour of nitrogen hydrate and the noble gas hydrates, it would be of great interest to explore these other diatomic element systems.

\section{Overview and conclusions}

The principal structural sequence amongst the gas hydrates that are cage-forming at low pressure is clear (see Fig. 12). Starting from a cubic clathrate structure at ambient pressure (either CS-I or CS-II), the first transformation on increasing pressure is to the hexagonal clathrate structure, SH. Although this structure appears to be common to almost all of the systems so far studied, we have seen that there may be large differences in the level of occupancy of the large cage. Shimizu and co-workers ${ }^{42}$ have suggested that there may even be phase transitions involving changes in the occupancy of this cage as a function of pressure. Detailed diffraction studies using singlecrystal techniques are needed to establish the levels of occupancy in the various systems. There is also a need to explore the effect of the preparation route and the composition of the starting material on the occupancy. The diffraction studies

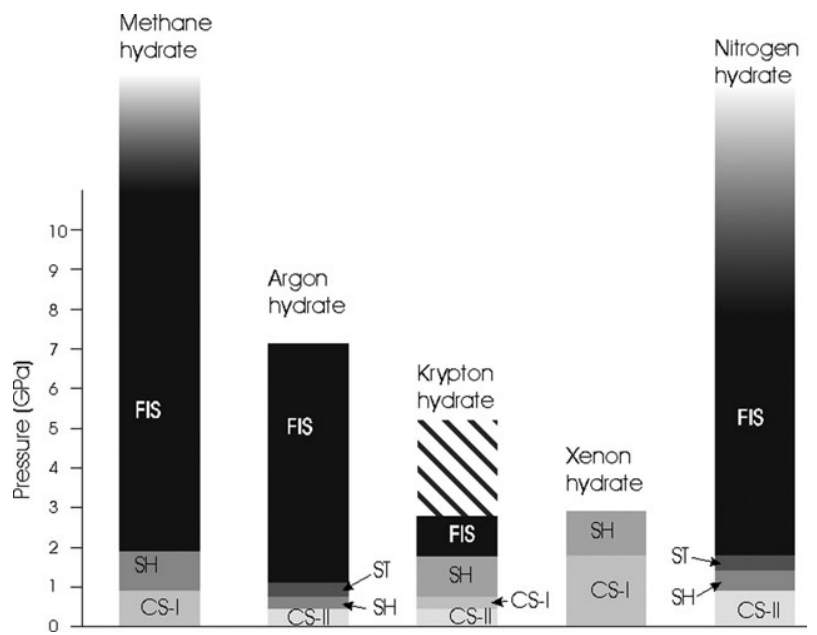

Fig. 12 The structural sequence adopted by the cage-forming gas hydrates whose structures have so far been explored. The notation 'CS-I', 'SH', 'FIS', etc., for the various hydrate structures is explained in section 2. The fade out of the bars for nitrogen hydrate and methane hydrate indicates that these two structures have not been observed to decompose up to the maximum pressure to which they have so far been studied, and the cross hatching for krypton hydrate represents the disagreement about the decomposition pressure discussed above in the text. have generally used samples loaded in the form of the lowpressure cage clathrate and then compressed. Shimizu et al. ${ }^{42}$ have tended to use samples loaded as a mixture of guest and host, from which they have grown single crystals in situ. Kuhs and co-workers ${ }^{10}$ have shown that the formation kinetics of classic cage clathrates can be very slow and that measured occupancies can be both time- and route-dependent, and it would not be surprising if this were true for the hexagonal structure as well.

It is also worth noting that much (though by no means all) of the work described here has explored the phase relations as a function of increasing pressure at constant temperature. It would clearly be valuable to establish phase relationships and transition lines by growing the various hydrate phases directly from the melt.

The post hexagonal-structure behaviour is somewhat less clear. It is tempting to associate the tetragonal structure (ST) with systems that adopt CS-II at low pressures. However, this structure is not observed in krypton hydrate. Our studies of argon and nitrogen hydrate suggest that the stability field of the tetragonal structure hydrate becomes very small close to room temperature because we observe samples transforming to and from this structure with small variations in the ambient temperature. It may be that krypton hydrate adopts the tetragonal structure at lower temperatures and a $P-T$ exploration of this and most of the other hydrate phase diagrams would be most useful.

The ultimate high-pressure structure adopted by all the systems which easily form cage clathrates (i.e. excepting $\mathrm{He}$, $\mathrm{H}_{2}$ and $\mathrm{Ne}$ ) is - with the notable exception of Xe hydrate - the $\mathrm{MH}-\mathrm{III}$ ice-I $h$-related filled-ice structure. As Shimizu et al. note, ${ }^{43}$ its $\mathrm{O}-\mathrm{H}$ stretch frequency and presumably its $\mathrm{H}$-bond geometry appear strongly dependent on the guest system unlike those of the classical clathrates and the MH-II structured hydrates. As a result it seems likely that there is a strong guest-host interaction and the stability of this structure is strongly dependent on the size of the guest. It is thus perhaps not surprising that xenon, which is the largest guest studied in detail, does not adopt this structure. Similarly one might expect that hydrogen, helium, and neon-even when starting from cage clathrate structures - would not adopt this structure since they are significantly smaller than the guests which do. It is not clear whether guest size plays a role in the ultimate high-pressure stability. Argon and nitrogen dihydrates have a similar guest size and yet very different stability ranges. However, it is interesting to note that extended stability above $10 \mathrm{GPa}$ has only been observed in polyatomic systems (methane and nitrogen). It is tempting to attribute extended stability to the additional entropy available from the rotational modes that are a feature of polyatomic guest molecules, but other polyatomic systems need to be studied.

\section{Acknowledgements}

We would like to thank D. D. Klug for helpful discussions and H. Shimizu and S. Desgreniers for access to currently unpublished data. We acknowledge the contribution of our colleagues and collaborators to work done by our group that is used in the review-including some unpublished results. Our work 
on clathrate hydrates is funded by the EPSRC and supported by STFC.

\section{References}

1 H. Davy, Philos. Trans. R. Soc. London, 1811, 101, 1-35.

2 V. R. Belosludov, M. Y. Lavrentiev, Y. A. Dyadin and S. A. Syskin, Izv. Akad. Nauk SSSR, Ser. Khim., 1989, 6, 49-56.

3 Y. A. Dyadin, E. Y. Aladko and E. G. Larionov, Mendeleev Commun., 1997, 7, 34-35.

4 S. L. Miller, Science, 1961, 134, 1431.

5 J. H. van der Waals and J. C. Platteeuw, Adv. Chem. Phys., 1959, 2, $1-57$.

6 J. I. Lunine and D. J. Stevenson, Astrophys. J., Suppl. Ser., 1985, 58, 493-531.

7 A. V. Milkov, Earth-Sci. Rev., 2004, 66, 183-197.

8 J. Maclennan and S. M. Jones, Earth Planet. Sci. Lett., 2006, 245, $65-80$.

9 J. H. Lee, Y. S. Baek and W. M. Sung, J. Ind. Eng. Chem., 2002, 8, 493-498.

10 B. Chazallon and W. F. Kuhs, J. Chem. Phys., 2002, 117, 308-320.

11 F. Pauer, S. Kipfstuhl, W. F. Kuhs and H. Shoji, J. Glaciol., 1999, 45, 22-30.

12 T. Hondoh, H. Anzai, A. Goto, S. Mae, A. Higashi and C. C. Langway, J. Inclusion Phenom. Mol. Recognit. Chem., 1990, 8, $17-24$.

13 J. Nakahara, Y. Shigesato, A. Higashi, T. Hondoh and C. C. Langway, Philos. Mag. B, 1988, 57, 421-430.

14 I. Chatti, A. Delahaye, L. Fournaison and J. P. Petitet, Energy Conv. Manage., 2005, 46, 1333-1343.

15 J. H. Lee, Y. S. Baek and W. M. Sung, Energy Sources, 2005, 27, $875-885$.

16 E. D. Sloan, Clathrate hydrates of natural gases, Marcel Dekker, New York, 2nd edn, 1998.

17 W. L. Vos, L. W. Finger, R. J. Hemley and H. K. Mao, Phys. Rev. Lett., 1993, 71, 3150-3153.

18 W. L. Mao, H. K. Mao, A. F. Goncharov, V. V. Struzhkin, Q. Z. Guo, J. Z. Hu, J. F. Shu, R. J. Hemley, M. Somayazulu and Y. S. Zhao, Science, 2002, 297, 2247-2249.

19 Y. A. Dyadin, E. G. Larionov, T. V. Mikina and L. I. Starostina, Mendeleev Commun., 1997, 7, 74-76.

20 Y. A. Dyadin, E. G. Larionov, D. S. Mirinski, T. V. Mikina and L. I. Starostina, Mendeleev Commun., 1997, 7, 32-34.

21 Y. A. Dyadin, E. G. Larionov, A. Y. Manakov, F. V. Zhurko, E. Y. Aladko, T. V. Mikina and V. Y. Komarov, Mendeleev Commun., 1999, 9, 209-210.

22 Y. A. Dyadin, E. Y. Aladko, A. Y. Manakov, F. V. Zhurko, T. V. Mikina, V. Y. Komarov and E. V. Grachev, J. Struct. Chem., 1999, 40, 790-795.

23 Y. A. Dyadin, E. G. Larionov, E. Y. Aladko and F. V. Zhurko, Dokl. Phys. Chem., 2001, 378, 159-161.

24 H. Hirai, M. Hasegawa, T. Yagi, Y. Yamamoto, K. Nagashima, M. Sakashita, K. Aoki and T. Kikegawa, Chem. Phys. Lett., 2000, 325, 490-498.

25 H. Hirai, T. Kondo, M. Hasegawa, T. Yagi, Y. Yamamoto, T. Komai, K. Nagashima, M. Sakashita, H. Fujihisa and K. Aoki, J. Phys. Chem. B, 2000, 104, 1429-1433.

26 H. Hirai, S. I. Machida, T. Kawamura, Y. Yamamoto and T. Yagi, Am. Mineral., 2006, 91, 826-830.

27 H. Hirai, T. Tanaka, T. Kawamura, Y. Yamamoto and T. Yagi, Phys. Rev. B, 2003, 68, 172102.

28 H. Hirai, T. Tanaka, T. Kawamura, Y. Yamamoto and T. Yagi, $J$. Phys. Chem. Solids, 2004, 65, 1555-1559.

29 H. Hirai, Y. Uchihara, H. Fujihisa, M. Sakashita, E. Katoh, K. Aoki, K. Nagashima, Y. Yamamoto and T. Yagi, J. Chem. Phys., 2001, 115, 7066-7070.

30 H. Hirai, Y. Uchihara, H. Fujihisa, M. Sakashita, E. Katoh, K. Aoki, Y. Yamamoto, K. Nagashima and T. Yagi, J. Phys.: Condens. Matter, 2002, 14, 11443-11446.

31 H. Hirai, Y. Uchihara, T. Kawamura, Y. Yamamoto and T. Yagi, Proc. Jpn. Acad. Ser. B: Phys. Biol. Sci., 2002, 78, 39-44.

32 H. Hirai, Y. Uchihara, Y. Nishimura, T. Kawamura, Y. Yamamoto and T. Yagi, J. Phys. Chem. B, 2002, 106, 11089-11092.
33 J. S. Loveday and R. J. Nelmes, High Pressure Res., 2003, 23, $41-47$.

34 J. S. Loveday, R. J. Nelmes and M. Guthrie, Chem. Phys. Lett., 2001, 350, 459-465.

35 J. S. Loveday, R. J. Nelmes, M. Guthrie, S. A. Belmonte, D. R. Allan, D. D. Klug, J. S. Tse and Y. P. Handa, Nature, 2001, 410, 661-663.

36 J. S. Loveday, R. J. Nelmes, M. Guthrie, D. D. Klug and J. S. Tse, Phys. Rev. Lett., 2001, 87, 215501.

37 J. S. Loveday, R. J. Nelmes and D. D. Klug, private communication, 2006.

38 J. S. Loveday, R. J. Nelmes, D. D. Klug, J. S. Tse and S. Desgreniers, Can. J. Phys., 2003, 81, 539-544.

39 S. Sasaki, S. Hori, T. Kume and H. Shimizu, J. Chem. Phys., 2003, 118, 7892-7897.

40 S. Sasaki, S. Hori, T. Kume and H. Shimizu, J. Phys. Chem. B, 2006, 110, 9838-9842.

41 H. Shimizu, Can. J. Phys., 2003, 81, 127-133.

42 S. Sasaki, T. Kume and H. Shimizu, in Physics and Chemistry of Ice (Proceedings of 11th International Conference on the Physics and Chemistry of Ice), ed. W. F. Kuhs, Royal Society of Chemistry, Cambridge, 2007, pp. 529-536.

43 H. Shimizu, S. Hori, T. Kume and S. Sasaki, Chem. Phys. Lett., 2003, 368, 132-138.

44 H. Shimizu, T. Kumazaki, T. Kume and S. Sasaki, Phys. Rev. B, $2002, \mathbf{6 5}, 212102$.

45 H. Shimizu, T. Kumazaki, T. Kume and S. Sasaki, J. Phys. Chem. $B, 2002,106,30-33$.

46 I. M. Chou, A. Sharma, R. C. Burruss, J. F. Shu, H. K. Mao, R. J. Hemley, A. F. Goncharov, L. A. Stern and S. H. Kirby, Proc. Natl. Acad. Sci. U. S. A., 2000, 97, 13484-13487.

47 Proceedings of the International Conference on Natural Gas Hydrates, New York Academy of Sciences, New York, 1993.

48 M. Von Stackelberg and H. R. Muller, J. Chem. Phys., 1951, 19, 1319-1320.

49 H. R. Muller and M. Von Stackelberg, Naturwissenschaften, 1952, 39, $20-21$

50 M. Von Stackelberg and H. R. Muller, Naturwissenschaften, 1951, 38, 456- 456 .

51 K. A. Udachin, C. I. Ratcliffe, G. D. Enright and J. A. Ripmeester, Supramol. Chem., 1997, 8, 173-176.

52 H. S. Kim and G. A. Jeffrey, J. Chem. Phys., 1970, 53, 3610-3615.

53 J. Baumert, C. Gutt, M. Johnson, J. Tse, D. Klug and W. Press, J. Chem. Phys., 2004, 120, 10163-10171.

54 K. Udachin, C. Ratcliffe and J. Ripmeester, J. Supramol. Chem., 2002, 2, 405-408.

55 G. Hummer, S. Garde, A. E. Garcia, M. E. Paulaitis and L. R. Pratt, Proc. Natl. Acad. Sci. U. S. A., 1998, 95, 1552-1555.

56 J. I. Lunine and D. J. Stevenson, Icarus, 1987, 70, 61-77.

57 A. Y. Manakov, V. I. Voronin, A. V. Kurnosov, A. E. Teplykh, E. G. Larionov and Y. A. Dyadin, Dokl. Phys. Chem., 2001, 378, $148-151$.

58 A. Y. Manakov, V. I. Voronin, A. V. Kurnosov, A. E. Teplykh, V. Y. Komarov and Y. A. Dyadin, J. Inclusion Phenom. Macrocyclic Chem., 2004, 48, 11-18.

59 T. Kumazaki, Y. Kito, S. Sasaki, T. Kume and H. Shimizu, Chem. Phys. Lett., 2004, 388, 18-22.

60 J. Baumert, C. Gutt, M. Krisch, H. Requardt, M. Muller, J. S. Tse, D. D. Klug and W. Press, Phys. Rev. B, 2005, 72, 054302.

61 D. D. Klug, J. S. Tse, Z. X. Liu and R. J. Hemley, J. Chem. Phys., 2006, 125, 154509.

62 T. Iitaka and T. Ebisuzaki, J. Phys.: Condens. Matter, 2004, 16, S1171-S1176.

63 T. Iitaka and T. Ebisuzaki, Phys. Rev. B, 2003, 68, 172105.

64 C. Sotin and G. Tobie, C. R. Phys., 2004, 5, 769-780.

65 G. Tobie, J. I. Lunine and C. Sotin, Nature, 2006, 440, 61-64.

66 E. R. Stofan, C. Elachi, J. I. Lunine, R. D. Lorenz, B. Stiles, K. L. Mitchell, S. Ostro, L. Soderblom, C. Wood, H. Zebker, S. Wall, M. Janssen, R. Kirk, R. Lopes, F. Paganelli, J. Radebaugh, L. Wye, Y. Anderson, M. Allison, R. Boehmer, P. Callahan, P. Encrenaz, E. Flamini, G. Francescetti, Y. Gim, G. Hamilton, S. Hensley, W. T. K. Johnson, K. Kelleher, D. Muhleman, P. Paillou, G. Picardi, F. Posa, L. Roth, R. Seu, S. Shaffer, S. Vetrella and R. West, Nature, 2007, 445, 61-64. 
67 A. Y. Manakov, S. V. Goryainov, A. V. Kurnosov, A. Y. Likhacheva, Y. A. Dyadin and E. G. Larionov, J. Phys. Chem. B, 2003, 107, 7861-7866.

68 E. Y. Aladko, A. I. Ancharov, S. V. Goryainov, A. V. Kurnosov, E. G. Larionov, A. Y. Likhacheva, A. Y. Manakov, V. A. Potemkin, M. A. Sheromov, A. E. Teplykh, V. I. Voronin and F. V. Zhurko, J. Phys. Chem. B, 2006, 110, 21371-21376.

69 Y. A. Dyadin, E. G. Larionov, A. Y. Manakov, A. V. Kurnosov, F. V. Zhurko, E. Y. Aladko, A. I. Ancharov, B. P. Tolochko and M. A. Sheromov, J. Inclusion Phenom. Macrocyclic Chem., 2002, 42, 213-218.

70 Y. P. Handa, J. S. Tse, D. D. Klug and E. Whalley, J. Chem. Phys., 1991, 94, 623-627.

71 O. Mishima, L. D. Calvert and E. Whalley, Nature, 1984, 310, 393-395.

72 J. S. Tse, in International Conference on Natural Gas Hydrates, ed. E. D. Sloan, J. Happel and M. A. Hnatow, New York Academy of Sciences, New York, 1994, vol. 715, pp. 187-206.

73 J. S. Tse, D. D. Klug, J. A. Ripmeester, S. Desgreniers and K. Lagarec, Nature, 1994, 369, 724-727.

74 H. Tanaka and Y. Amano, Mol. Phys., 2002, 100, 2183-2188.

75 Y. Suzuki, Phys. Rev. B, 2004, 70, 172108.

76 Y. A. Dyadin, E. G. Larionov and A. Y. Manakov, Gas hydrates at high pressures - State of the art, in Proceedings of the Fourth International Conference on Gas Hydrates, ICGH Yokohama, Yokohama, 2002, pp. 590-594.

77 D. W. Davidson, M. A. Desando, S. R. Gough, Y. P. Handa, C. I. Ratcliffe, J. A. Ripmeester and J. S. Tse, Nature, 1987, 328, 418-419.
78 A. Chapoy, A. H. Mohammadi, B. Tohidi, A. Valtz and D. Richon, Ind. Eng. Chem. Res., 2005, 44, 7567-7574.

79 D. Londono, W. F. Kuhs and J. L. Finney, Nature, 1988, 332, 141-142.

80 D. Londono, J. L. Finney and W. F. Kuhs, J. Chem. Phys., 1992, 97, 547-552.

81 C. Lobban, J. L. Finney and W. F. Kuhs, J. Chem. Phys., 2002, 117, 3928-3934.

82 H. T. Lotz and J. A. Schouten, J. Chem. Phys., 1999, 111, 10242-10247.

83 A. V. Kurnosov, A. Y. Manakov, V. Y. Komarov, V. I. Voronin, A. E. Teplykh and Y. A. Dyadin, Dokl. Phys. Chem., 2001, 381, 303-305.

84 D. R. Marschall, S. H. Saito and R. Kobayashi, AIChE J., 1964, 10, 22.

85 H. Itoh, J. S. Tse and K. Kawamura, J. Chem. Phys., 2001, 115, 9414-9420.

86 S. Alavi, J. A. Ripmeester and D. D. Klug, J. Chem. Phys., 2006, 125, 104501.

87 S. Desgreniers, private communication, 2004.

88 C. Sanloup, H. K. Mao and R. J. Hemley, Proc. Natl. Acad. Sci. U. S. A., 2002, 99, 25-28.

89 W. L. Vos, L. W. Finger, R. J. Hemley and H. K. Mao, Chem. Phys. Lett., 1996, 257, 524-530.

90 M. G. E. Van Hinsberg, M. I. M. Scheerboom and J. A. Schouten, J. Chem. Phys., 1993, 99, 752-754.

91 S. Machida, H. Hirai, T. Kawamura, Y. Yamamoto and T. Yagi, Phys. Earth Planet. Interiors, 2006, 155, 170-176. 